\title{
The FgVps39-FgVam7-FgSso1 Complex Mediates Vesicle Trafficking and Is Important for the Development and Virulence of Fusarium graminearum
}

\author{
Bing Li, ${ }^{1}$ Luping Liu, ${ }^{1}$ Ying Li, ${ }^{1}$ Xin Dong, ${ }^{1}$ Haifeng Zhang, ${ }^{1}$ Huaigu Chen, ${ }^{2}$ Xiaobo Zheng, ${ }^{1}$ and \\ Zhengguang Zhang ${ }^{1}$ \\ ${ }^{1}$ Department of Plant Pathology, College of Plant Protection, Nanjing Agricultural University, and Key Laboratory of Integrated \\ Management of Crop Diseases and Pests, Ministry of Education, Nanjing 210095, China; and ${ }^{2}$ Institute of Plant Protection, \\ Jiangsu Academy of Agricultural Sciences, Nanjing 210014, China
}

Accepted 8 March 2017.

\begin{abstract}
Vesicle trafficking is an important event in eukaryotic organisms. Many proteins and lipids transported between different organelles or compartments are essential for survival. These processes are mediated by soluble $\mathrm{N}$-ethylmaleimide-sensitive factor attachment protein receptor (SNARE) proteins, RabGTPases, and multisubunit tethering complexes such as class $\mathbf{C}$ core vacuole or endosome tethering and homotypic fusion or vacuole protein sorting (HOPS). Our previous study has demonstrated that FgVam7, which encodes a SNARE protein involving in vesicle trafficking, plays crucial roles in growth, asexual or sexual development, deoxynivalenol production, and pathogenicity in Fusarium graminearum. Here, the affinity purification approach was used to identify FgVam7-interacting proteins to explore its regulatory mechanisms during vesicle trafficking. The orthologs of yeast Vps39, a HOPS tethering complex subunit, and Sso1, a SNARE protein localized to the vacuole or endosome, were identified and selected for further characterization. In yeast two-hybrid and glutathione-S-transferase pull-down assays, FgVam7, FgVps39, and FgSso1 interacted with each other as a complex. The $\Delta F g v p s 39$ mutant generated by targeted deletion was significantly reduced in vegetative growth and asexual development. It failed to produce sexual spores and was defective in plant infection and deoxynivalenol production. Further cellular localization and cytological examinations suggested that FgVps39 is involved in vesicle trafficking from early or late endosomes to vacuoles in $F$. graminearum. Additionally, the $\Delta F g v p s 39$ mutant was defective in vacuole morphology and autophagy, and it was delayed in endocytosis. Our results demonstrate that FgVam7 interacts with $\mathrm{FgVps39}$ and FgSso1 to form a unique complex, which is involved in vesicle trafficking and modulating the proper development of infection-related morphogenesis in $F$. graminearum.
\end{abstract}

Soluble N-ethylmaleimide-sensitive factor attachment protein receptor (SNARE) proteins are a large family of proteins that play a central role in membrane fusion and vesicle

Corresponding author: H. Zhang; Telephone: 86-25-84396436; Fax: 86-25-84396436; E-mail: hfzhang@njau.edu.cn

*The $e$-Xtra logo stands for "electronic extra" and indicates that three supplementary figures, two supplementary tables, and nine supplementary movies are published online.

(C) 2017 The American Phytopathological Society trafficking of eukaryotic organisms (Dou et al. 2011; Qi et al. 2016; Rothman 1994). SNARE proteins share a conserved structure called the SNARE motif (Fasshauer et al. 1998; Jahn et al. 2003), and they have been reported to play important roles in the development and virulence of mammals and fungi (Burri and Lithgow 2004; Burri et al. 2003; Dou et al. 2011; Qi et al. 2016; Song et al. 2010). In Drosophila spp., SNARE protein Snap29 localizes to multiple trafficking organelles, and it is required for vesicle trafficking and epithelial formation (Morelli et al. 2014). In the rice blast fungus Magnaporthe oryzae, SNARE proteins MoVam7 and MoSec22 mediate endocytosis and vacuole fusion, and play crucial roles in vegetative growth, conidiogenesis, and virulence (Dou et al. 2011; Song et al. 2010). Another SNARE protein (MoSyn8) localizes to endosomes and mediates endocytosis and secretion, thus controlling the development of infectious hyphal growth (Qi et al. 2016).

In Fusarium graminearum (teleomorph: Gibberella zeae), the causal agent of Fusarium head blight or scab disease in various cereal crops (Bai et al. 2002; Goswami and Kistler 2004; McMullen et al. 1997), the SNARE protein FgVam7 was recently found to play important roles in vegetative growth, asexual or sexual development, deoxynivalenol (DON) production, and pathogenicity by mediating vesicle trafficking and vacuole fusion (Zhang et al. 2016). However, it is not clear how FgVam7 mediates vesicle trafficking and vacuole fusion, and which proteins function together with FgVam7 during these processes.

In Saccharomyces cerevisiae, Vam7 interacts with Vps39, a member of the homotypic fusion or vacuole protein sorting (HOPS) complex that is involved in the endosome to vacuole transportation (Balderhaar and Ungermann 2013). Vesicle trafficking transports proteins and lipid between different organelles or compartments and plays important roles in the development of eukaryotic cells. This process mainly depends on membrane fusion events mediated by SNARE proteins, Rab GTPases, and multisubunit tethering complexes (van der Kant et al. 2015), including class $\mathrm{C}$ core vacuole or endosome tethering complex, HOPS complex, the exocyst at plasma membrane, the transport protein particle family, and the conserved oligomeric Golgi complex at the Golgi (Balderhaar and Ungermann 2013; Peplowska et al. 2007; Seals et al. 2000). The HOPS tethering complex contains six subunits, including Vps11, Vps16, Vps18, Vps33, Vps39, and Vps41 (Rothman and Stevens 1986; Wartosch et al. 2015). Among them, Vps11, Vps16, Vps18, and Vps33 are core components of the HOPS complex and play crucial roles in endosomal biogenesis and vacuole morphology (Balderhaar and Ungermann 2013). Subunits Vps39 and Vps41 
that bind to Rab7 were involved in vacuolar morphogenesis (Balderhaar and Ungermann 2013). As a guanine nucleotide exchange factor (GEF) of Gtr1 (Wurmser et al. 2000), Vps39 is known to activate Gtr1 to control TORC1 activity in S. cerevisiae (Binda et al. 2009; Li and Guan 2009; Valbuena et al. 2012). Vps39 orthologs have been characterized in a few fungal pathogens (Oka et al. 2004; Ramanujam et al. 2013; Yang et al. 2016). In $M$. oryzae, MoVps39 is essential for appressorium formation and pathogenicity. In Coniothyrium minitans, $\mathrm{CmVps} 39$ is indispensable for vacuolar morphology, autophagy, conidiogenesis, and mycoparasiticism (Yang et al. 2016).

To further characterize the functions of FgVam7 in vesicle trafficking and vacuole fusion, in this study we used the affinity purification approach to identify FgVam7-interacting proteins in $F$. graminearum. Orthologs of the vacuole-sorting protein Vps39 and the SNARE protein Sso1 were among the FgVam7interacting proteins identified and were selected for further characterization. Our data showed that FgVam7, FgVps39, and FgSso1 function as a protein complex during vesicle trafficking, endocytosis, and autophagy, thus controlling development, plant infection, and DON production in F. graminearum. These results not only help to uncover the regulatory mechanism of FgVam7 but also benefit our understanding of the functions of SNARE proteins and vesicle trafficking during fungal development and pathogenesis.

\section{RESULTS}

Identification of proteins associated with $\mathrm{FgVam} 7$ in vivo.

Our previous study showed that the SNARE protein FgVam7 (FGSG_12950, EYB28243.1) was involved in vegetative growth, conidiation, sexual reproduction, DON production, virulence, vacuole fusion, and endocytosis in $F$. graminearum (Zhang et al. 2016). To identify FgVam7-interacting proteins by affinity purification (Zhou et al. 2012), we generated the FgVAM7-3×FLAG fusion construct and transformed it into the wild-type strain PH-1. A protein band with the expected size of FgVam7-3×FLAG was detected in Western blots of proteins isolated from the resulting transformants. Proteins bound to the anti-Flag M2 beads were eluted following affinity purification and analyzed by mass spectrometry analysis (Supplementary Table S1). Two of the FgVam7interacting proteins, FgVps39 (FGSG_12272, XP_011321275.1) and FgSso1 (GzSyn1: FGSG_00950, XP_011316692.1), are highly similar to yeast Vps39 and Sso1 that are involved in vesicle trafficking (Aalto et al. 1993; Balderhaar and Ungermann 2013). Other proteins that coimmunoprecipitated with FgVam7 include GTPbinding associated proteins and a number of hypothetical proteins in F. graminearum. FgVps39 is an 891-amino-acid protein with an N-terminal citron homology domain and a C-terminal Vps39-1 domain. Its orthologs are well conserved in fungi and it shares $50 \%$ amino acid similarity to Neurospora crassa NcVps39 (Supplementary Fig. S1). FgSso1 is a SNARE protein that is known to be involved in the development and pathogenesis of $F$. graminearum (Hong et al. 2010).

\section{FgVps39, FgSso1, and FgVam7 physically interact with each other as a complex in $F$. graminearum.}

To confirm the relationship among FgVps39, FgVam7, and FgSso1, we performed yeast two-hybrid (Y2H) and in vitro glutathione S-transferase (GST) pull-down assays. The cDNA of $\mathrm{FgVPS} 39$ and $\mathrm{FgSSO} 1$ was cloned into pGADT7 as the prey vector and the cDNA of $\mathrm{FgVAM7}$ and $\mathrm{FgSSOl}$ was cloned into pGBKT7 as the bait vector. The resulting bait and prey constructs were cotransformed in pairs into AH109 yeast cells (Schiestl and Gietz 1989). The $\mathrm{Leu}^{+}$and $\mathrm{Trp}^{+}$transformants were isolated and assayed for growth on synthetic medium lacking trytophan, leucine, histidine, and adenine (SD-Trp-Leu-His-Ade medium) or SD-Trp-Leu-His medium. The 3-aminotyiazole (3AT) was used to assess the binding activity (Cohen and Schuldiner 2011). The results showed that FgVps39, FgVam7, and FgSso1 interacted with each other in $\mathrm{Y} 2 \mathrm{H}$ assays (Fig. 1A, C, and E). Their interactions were further verified by GST pull-down assays. The cDNA of $\mathrm{FgVPS} 39, \mathrm{FgVAM} 7$, and $\mathrm{FgSSO} 1$ were cloned into the expression vector pGEX-4T-2 or pET-32a. The resulting constructs GST-FgVps39, GST-FgSso1, His-FgVps39, and HisFgVam7 were transformed into Escherichia coli strain BL21. Lysates of GST-FgVps39 or GST-FgSso1 transformants or GST were incubated with lysates of His-FgVam7 or His-FgVps39 transformants, and applied to glutathione GST-binding resin. GST-FgVps39- and GST-FgSso1-bound resins but not GSTbound resins provided enrichment of His-FgVps39 and HisFgVam7 (Fig. 1B, D, and F). These results suggest that FgVps39, FgSso1, and FgVam7 interact with each other and likely function as a complex in $F$. graminearum.

\section{FgVps39 localizes to early and late endosomes in $F$. graminearum.}

To investigate the role of $\mathrm{FgVps} 39$, we first checked the subcellular localization of the FgVps39 protein in F. graminearum. The GFP-FgVps39 fusion construct driven by its native promoter was generated and transformed into the $\Delta F$ gvps 39 mutant. Because the resulting transformants displayed weak green fluorescent protein (GFP) signals, we used the strong constitutive ribosomal protein promoter $R P 27$ to express the GFP-FgVps39 fusion and obtained transformants with strong GFP signals under a confocal fluorescence microscope. Most of the GFP signals were distributed in vacuoles and a few were observed in punctate structures (Fig. 2A). After 2 to $4 \mathrm{~h}$ of incubation in YEPD medium ( $3 \mathrm{~g}$ of yeast extract, $10 \mathrm{~g}$ of peptone, $20 \mathrm{~g}$ of dextrose in 1 liter of distilled water) for germination, GFP signals were transformed from the vacuole to punctate structures. The number of punctate structures with GFP signals increased $2 \mathrm{~h}$ postinoculation (hpi), and almost all GFP was observed in the punctate structures in conidia and germ tubes 4 hpi (Fig. 2A). GFP signals again accumulated in vacuoles in conidia and primary germ tubes $6 \mathrm{hpi}$ but with punctate structures in new germ tubes even at 24 hpi (Fig. 2A). Because yeast Vps39 is involved in vesicle trafficking from late endosomes to the vacuole (Balderhaar and Ungermann 2013), we stained the conidia and germ tubes with FM4-64 (time-dependent dye that does not distinguish between early and late endosomal compartmentalization) and 7-amino-4-chloromethylcoumarin (CMAC). The results showed that the GFP punctate structures merged well with the FM4-64-stained endosomes and vacuole (Fig. 2A), indicating that FgVps39 localized in the vacuole and endosomes. Further linescan graph analysis data supported these colocalization results (Fig. 2B and C). Together with the previous results that $\mathrm{FgVam} 7$ localized in the vacuole and FgSso1 in the vacuole and endosomes (Hong et al. 2010; Zhang et al. 2016), we conclude that FgVps39-FgVam7-FgSso1 functions as a complex to mediate vesicle trafficking in $F$. graminearum.

To determine whether FgVps39 localized in early or late endosomes, we used the early- and late-endosome-specific markers FgRab51 and FgRab7 for colocalization analysis with $\mathrm{FgVps} 39$. We found that $\mathrm{FgVps} 39$ colocalized with FgRab51 and FgRab7 in most cases (Fig. 3A and B; Supplementary Movies S1, S2, S3, S4, S5, and S6). We concluded that $\mathrm{FgVps} 39$ is involved in vesicle trafficking of the early and late endosomes to the vacuole in $F$. graminearum.

\section{The movement of $\mathrm{FgVps39}$ protein is facilitated by the microtubular cytoskeleton.}

FgVps39-red fluorescent protein (RFP) in punctate structures appeared to be short range, fast moving, and highly dynamic in the hyphae. Transportation activities in eukaryotic cells are facilitated by the microtubules, and the actin cytoskeleton is a 
key component involved in these processes (Ramanujam et al. 2013). Microtubules are formed by polymerization of $\alpha$ - and $\beta$-tubulin dimers (Desai and Mitchison 1997; Luo et al. 2014). To investigate whether the fast movement of FgVps39-RFP depends on microtubules or the actin cytoskeleton, a transformant that coexpressed GFP-FgVps39 and $\beta$-tubulin tagged with mCherry (Tub2-mCherry) or LifeAct-GFP constructs was generated. The resulting transformants showed that FgVps39 associated with microtubules in the hyphae (Fig. 4). The movement of FgVps39 along tubulin filaments is shown in Supplementary Movie S7. In contrast, no apparent colocalized fluorescence was observed in the hyphae of the FgVps39-RFP and LifeAct-GFP coexpressing strain (Supplementary Fig. S2), indicating that the fast movement of FgVps39-RFP depended on the microtubular cytoskeleton but not the F-actin cytoskeleton.

In order to clarify how this fast movement depended on the microtubular cytoskeleton, we observed GFP-FgVps39 movement in hyphae treated with microtubule destabilizing agent nocodazole (Kerr et al. 2006; Moisoi et al. 2002). Compared with movement in the dimethyl sulfoxide control treatment (Supplementary Movie S8), movement of the GFP-FgVps39 punctate compartments was highly restricted after the nocodazole treatment (Supplementary Movie S9). These findings demonstrate that the movements of GFP-FgVps39 are facilitated by the $F$. graminearum microtubular cytoskeleton.

\section{FgVps39 is required for normal hyphal growth,} conidiogenesis, and female fertility.

To investigate the roles of $\mathrm{FgVps} 39$ in $F$. graminearum, an FgVPS39 gene deletion vector was generated by a splitmarker approach (Supplementary Fig. S3A) and transformed into protoplasts of the wild-type $\mathrm{PH}-1$. The resulting transformants were screened by polymerase chain reaction (PCR) and further confirmed by Southern blot analysis. The complemented transformant $\triangle F g v p s 39 / F g V P S 39$ was generated by reintroducing the full-length FgVPS39 into the $\Delta$ Fgvps39 mutant.

To explore the role of FgVps39 in vegetative growth and asexual development, wild-type PH-1, the $\Delta$ Fgvps 39 mutant, and the complemented transformant $\Delta F g v p s 39 / F g V P S 39$ were cultured on $\mathrm{V} 8,5 \times$ YEG (5 $\mathrm{g}$ of yeast extract, $10 \mathrm{~g}$ of D-glucose in 1 liter of distilled water), complete medium (CM), minimal medium (MM) agar plates. After 3 days of incubation, the $\Delta F g v p s 39$ mutant exhibited a significantly reduced growth rate with fewer aerial hyphae on these four media (Fig. 5A; Table 1). We also quantified conidial production of the $\Delta F g v p s 39$ mutant in carboxymethyl cellulose (CMC) medium. After a 5-day incubation on a shaker, $\Delta$ Fgvps 39 exhibited a significant decrease in conidiation (Fig. 5B; Table 1), and showed detectable changes in conidial morphology, because $81.2 \%$ of the conidia had constricted septa compared with those of the PH-1 wild type (Fig. 5C). These results indicate that $\Delta F g v p s 39$ has a role in vegetative growth and asexual development in $F$. graminearum. Because ascospores are important for the disease cycle of $F$. graminearum, we analyzed sexual development of the $\Delta F g v p s 39$ mutant, as described previously (Zhang et al. 2016). Ten days after inoculation, the $\Delta$ Fgvps39 mutant failed to produce perithecia in comparison with numerous mature perithecia produced by $\mathrm{PH}-1$ and the complemented strains under the same conditions (Fig. 5D), indicating that FgVps39 plays an essential role in sexual reproduction in $F$. graminearum.

\section{FgVps39 plays critical roles in virulence and DON production in $\boldsymbol{F}$. graminearum.}

To determine the involvement of $\mathrm{FgVps} 39$ in virulence, we first assayed the $\Delta F$ gvps 39 mutant on flowering wheat heads. Compared with the wild-type $\mathrm{PH}-1$ that caused obvious head blight symptoms, the $\Delta F g v p s 39$ mutant caused no symptoms 14 days postinoculation (Fig. 6A). We further tested the
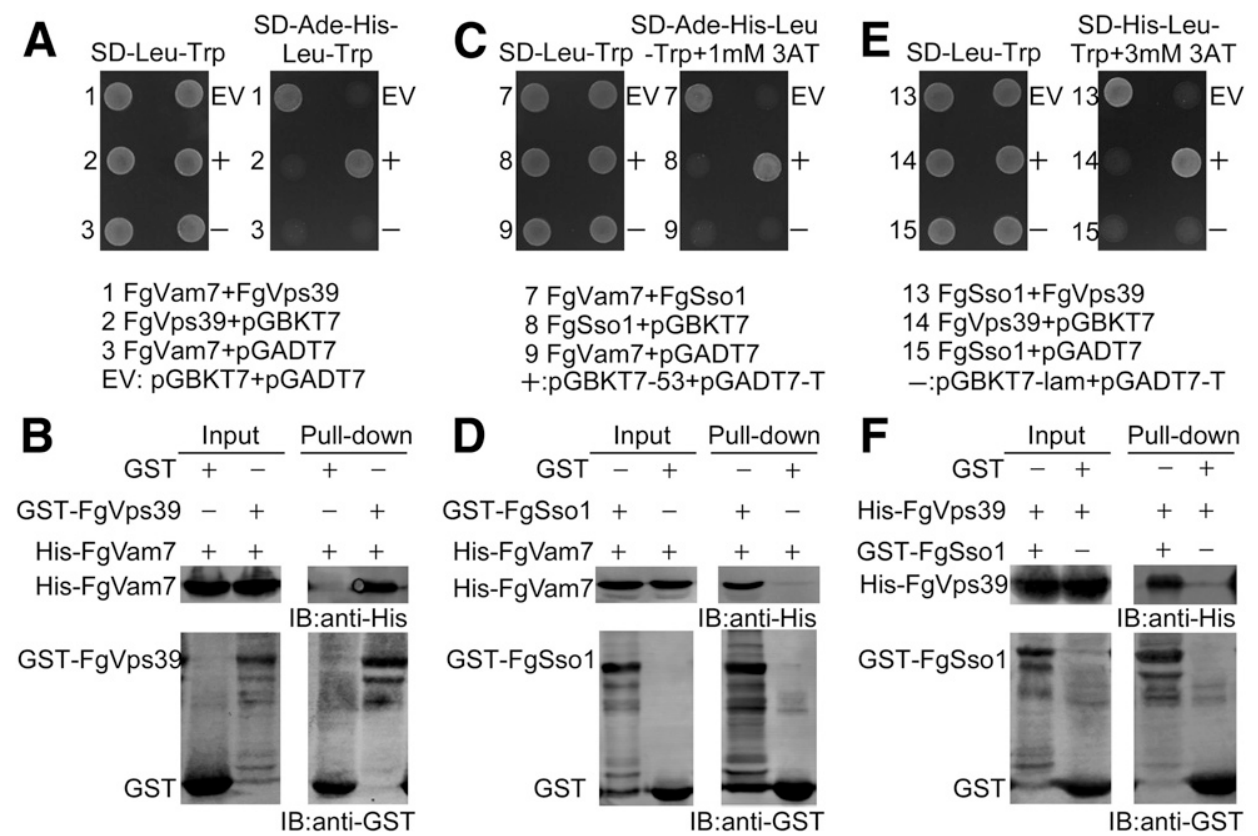

Fig. 1. Yeast two-hybrid and glutathione-S-transferase (GST) pull-down assays. A, C, and E, Yeast two-hybrid assay for the interaction among FgVps39, FgSso1, and FgVam7. Full-length $\mathrm{FgVPS} 39$ and $\mathrm{FgSSO} 1$ were inserted into the vector pGADT7 (AD) and full-length $\mathrm{FgVAM7}$ and $\mathrm{FgSSO} 1$ were inserted into the vector pGBKT7 (BD). The AD and BD plasmids were cotransformed into yeast AH109 cells, and plated on synthetic medium lacking leucine and tryptophan (SD-Leu-Trp) for 3 days or on selective medium lacking Leu, Trp, histidine, and adenine (SD-Leu-Trp-His-Ade) with or without 3-aminotyiazole (3AT) for 3 days. B, D, and F, GST pull-down assays for FgVps39, FgVam7, and FgSso1. His-Vps39, His-Vam7, GST-Vps39, and GST-Sso1 were expressed and purified by affinity chromatography. The eluted protein was analyzed by immunoblot (IB) with anti-His and anti-GST antibodies. Bound proteins were separated by sodium dodecyl sulfate polyacrylamide gel electrophoresis in duplicate and analyzed by Western blot. 
infection on maize stalks and wheat germlings by droplet inoculation. The $\Delta$ Fgvps 39 mutant caused only limited discoloration and failed to spread at the inoculation site, whereas extensive disease symptoms were observed on maize stalks inoculated with $\mathrm{PH}-1$ and the complemented strains under the same conditions (Fig. 6B). Similar results were observed on wheat germ (Fig. 6C). A microscopic examination revealed that the mutant was unable to penetrate through the wheat coleoptile epidermis and no invasive hyphae were found in plant cells, whereas both the wild-type PH-1 and the complemented strains
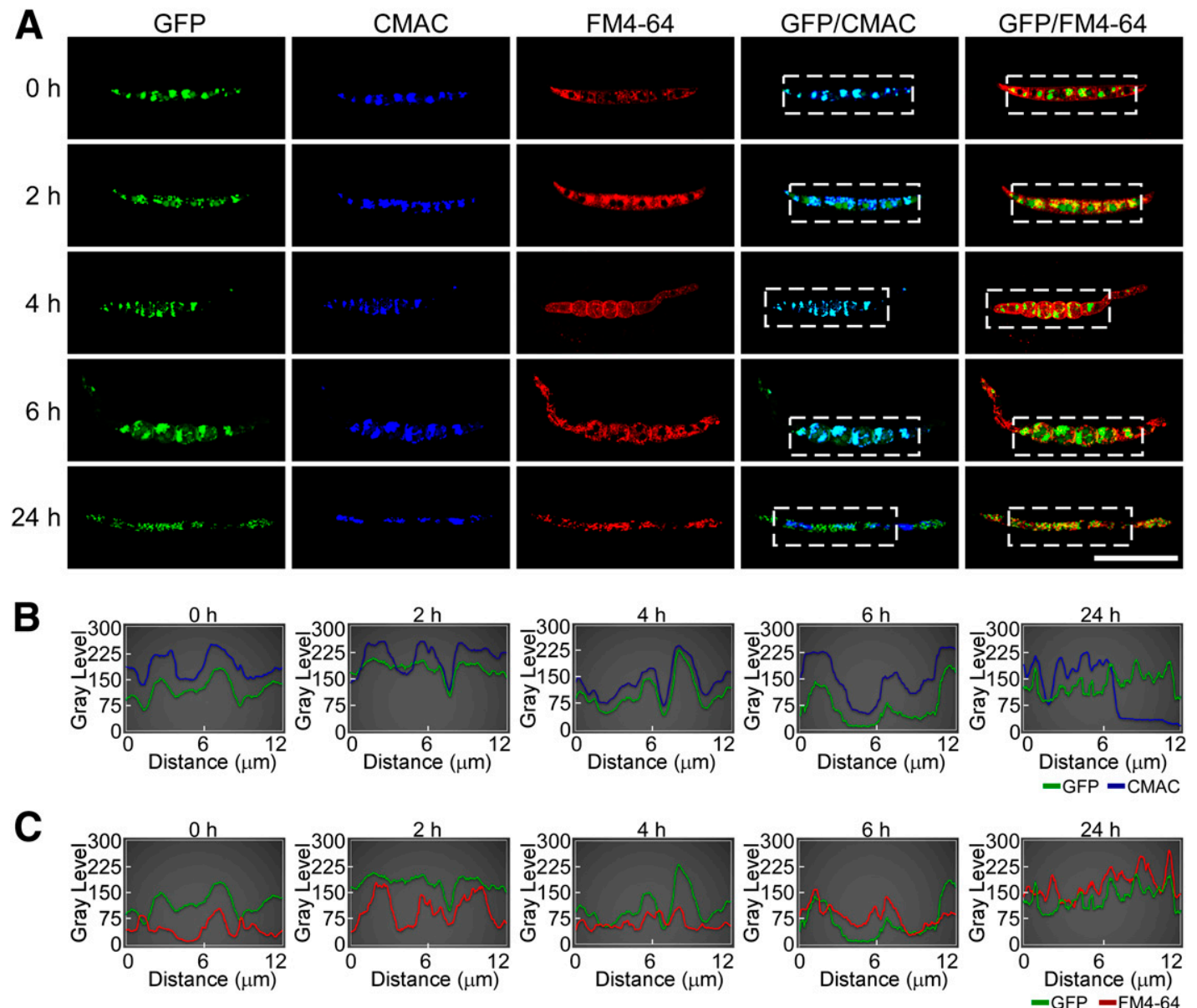

Fig. 2. Expression and subcellular localization of green fluorescent protein (GFP)-FgVps39 in conidia and germ tubes. A, Conidia expressing the GFPFgVPS39 fusion construct were harvested and germinated in liquid complete medium for 0, 2, 4, 6, and $24 \mathrm{~h}$, and photographed. Conidia and germ tubes were stained with FM4-64 and 7-amino-4-chloromethylcoumarin (CMAC) and examined by fluorescence microscopy. GFP/CMAC indicates merged GFP and CMAC images. GFP/FM4-64 indicates merged GFP and FM4-64 images. Bar $=20 \mu \mathrm{m}$. B, Linescan graph consistent with colocalization of GFP-FgVps39 and CMAC. C, Linescan graph consistent with colocalization of GFP-FgVps39 and FM4-64.
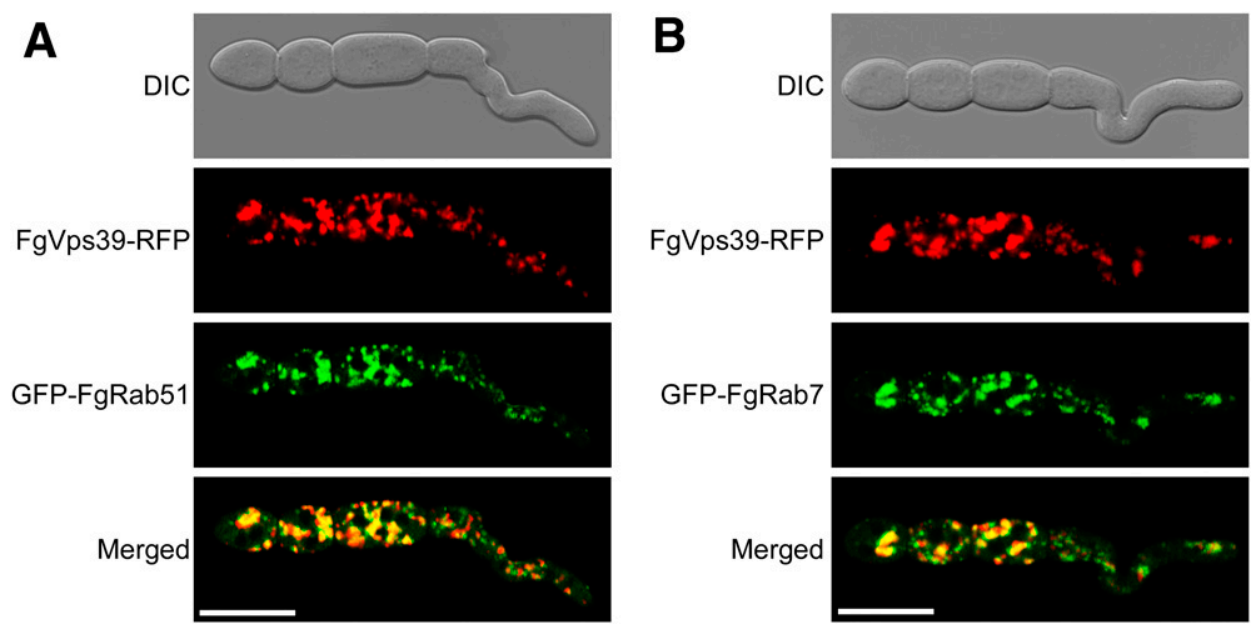

Fig. 3. FgVps 39 localizes to both early and late endosomes in Fusarium graminearum. A, Conidial germination $(4 \mathrm{~h})$ of $F$. graminearum coexpressing green fluorescent protein (GFP)-FgRab51 and FgVps39-red fluorescent protein (RFP) was observed under confocal microscopy. B, Conidial germination (4 h) of $F$. graminearum coexpressing GFP-FgRab7 and FgVps39-RFP was observed under fluorescence confocal microscopy. Bar $=10 \mu \mathrm{m}$. DIC, differential interference contrast microscopy. 
formed branching and expanded infectious hyphae in cells (Fig. 6D).

DON was reported to relate to virulence in $F$. graminearum (Desjardins et al. 1996; Proctor et al. 1995; Seong et al. 2009). DON production was assayed in wheat kernels infected with $\mathrm{PH}-1$ and the $\Delta$ Fgvps 39 mutant. DON production was very low in the $\Delta$ Fgvps39 mutant, with DON at only $0.013 \mathrm{mg} / \mathrm{mg}$ of

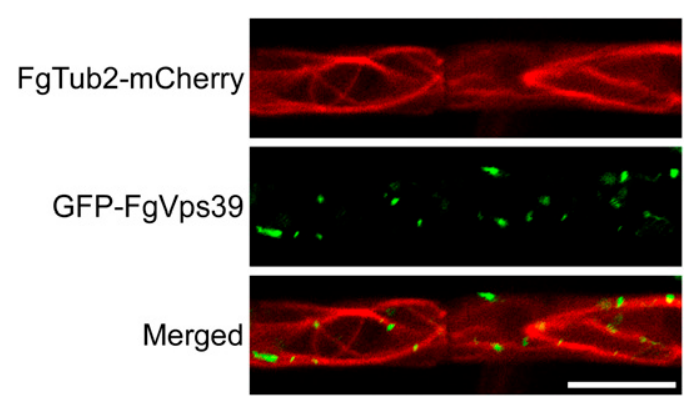

Fig. 4. Close associations between green fluorescent protein (GFP)FgVps39 and microtubule cytoskeleton. Hyphae coexpressing GFPFgVps39 and FgTub2-mCherry were observed under fluorescence confocal microscopy. Bar $=10 \mu \mathrm{m}$. ergosterol, whereas DON was detected at more than $4 \mathrm{mg} / \mathrm{mg}$ of ergosterol in the wild-type PH-1 (Table 1). To further confirm this result, we determined the transcription levels of the DON biosynthesis-related genes TRI1, TRI3, TRI5, TRI6, TRI12, and TRI101 by quantitative real-time (qRT)-PCR. The transcription levels of these six genes decreased remarkably in the $\Delta$ Fgvps39 mutant (Fig. 6E). Collectively, these results indicate that FgVps39 controls DON biosynthesis by modulating the expression of TRI genes and eventually affecting DON synthesis in $F$. graminearum.

\section{Deletion of FgVps39 delays endocytosis in $\boldsymbol{F}$. graminearum.}

Vps39 is involved in vesicle trafficking from the late endosomes to vacuolar assembly of the cytoplasm-to-vacuole targeting (CVT) pathway in S. cerevisiae (Balderhaar and Ungermann 2013; Nakamura et al. 1997). FM4-64 specifically binds to the plasma membrane and enters the vacuole through endocytosis (Fischer-Parton et al. 2000; Vida and Emr 1995). We examined endocytosis of the $\Delta F g v p s 39$ mutant by FM4-64 staining. Wild-type PH-1 and the complemented transformant took up the FM4-64 dye within 1 min of exposure, and numerous intact endosomes were stained and observed in the hyphae. However, no fluorescent signal was observed in $\Delta$ Fgvps39
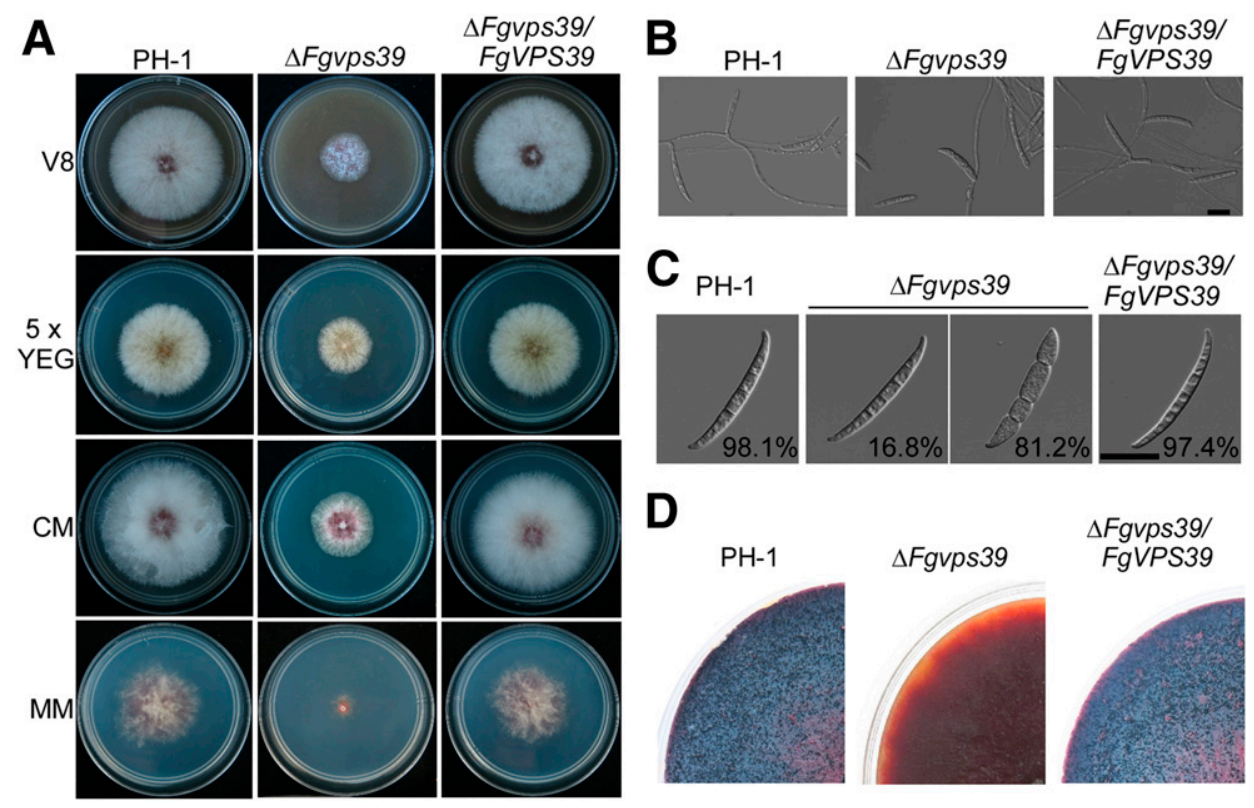

Fig. 5. Vegetative growth and asexual or sexual development defects of the $\Delta$ Fgvps 39 mutant. A, Colony of the wild-type PH-1, the $\Delta$ Fgvps 39 mutant, and the complemented transformant $\triangle F g v p s 39 / F g V P S 39$ on different media. Photographs were taken at 3 days after incubation (dai). $5 \times$ YEG $=$ yeast extract and glucose medium, $\mathrm{CM}=$ complete medium, and $\mathrm{MM}=$ minimal medium. $\mathbf{B}$ and $\mathbf{C}$, Conidial production and morphology of PH-1, $\Delta F g v p s 39$, and $\Delta$ Fgvps39/FgVPS39 in 5-day-old carboxymethyl cellulose cultures. Bar $=10 \mu \mathrm{m}$. D, Sexual reproduction of the indicated strains on carrot media and photographed at 10 dai. The black ball-like structures are perithecia.

Table 1. Phenotype analysis of the wild type, $\Delta F g v p s 39$, and $\Delta F g v p s 39 / F g V P S 39$ transformant in Fusarium graminearum ${ }^{\mathrm{a}}$

\begin{tabular}{|c|c|c|c|c|c|c|}
\hline \multirow[b]{2}{*}{ Strain } & \multicolumn{4}{|c|}{ Colony diameter $(\mathbf{c m})^{b}$} & \multirow[b]{2}{*}{ Con $\left(\times 10^{6} / \mathrm{ml}\right)^{\mathrm{c}}$} & \multirow[b]{2}{*}{$\operatorname{DON}(\%)^{\mathrm{d}}$} \\
\hline & $\mathbf{C M}$ & V8 & 5XYEG & MM & & \\
\hline PH-1 & $7.2 \pm 0.2$ & $5.7 \pm 0.1$ & $5.4 \pm 0.1$ & $5.0 \pm 0.1$ & $1.34 \pm 0.2$ & $4.17 \pm 0.10$ \\
\hline$\Delta F g v p s 39$ & $3.4 \pm 0.1^{*}$ & $2.9 \pm 0.1 *$ & $2.7 \pm 0.1^{*}$ & $1.3 \pm 0.1^{*}$ & $0.09 \pm 0.06^{*}$ & $0.013 \pm 0.001 *$ \\
\hline$\Delta$ Fgvps39/FgVPS39 & $7.2 \pm 0.1$ & $5.8 \pm 0.1$ & $5.4 \pm 0.1$ & $4.9 \pm 0.1$ & $1.29 \pm 0.1$ & NA \\
\hline
\end{tabular}


mutant hyphal cells after $1 \mathrm{~min}$. The fluorescent signal could be observed on the plasma membrane after $2 \mathrm{~min}$ and at the endosomal compartments after $3 \mathrm{~min}$ (Fig. 7A and B). These results suggest that $\mathrm{FgVps} 39$ plays an important role in endocytosis.

\section{FgVps39 binds the GTP-associated version} of FgRab7 rather than GDP version in vitro.

GTPase Rab7 regulates the HOPS complex during late endosomes to the vacuole in yeast (Balderhaar and Ungermann 2013). GTPase FgRab7 is indispensable for membrane traffickingdependent growth and plant infection in $F$. graminearum (Zheng et al. 2015). To clarify the relationship between FgVps39 and FgRab7, we constructed vectors containing a GTP-associated version of $\mathrm{FgRab} 7^{\mathrm{Q} 67 \mathrm{~L}}$ and a GDP-associated version of $\mathrm{FgRab} 7^{\mathrm{T} 22 \mathrm{~N}}$ and assayed them in the $\mathrm{Y} 2 \mathrm{H}$ assay and in vitro GST pull-down assay. The results showed that the interaction between $\mathrm{FgVps} 39$ and $\mathrm{FgRab} 7^{\mathrm{Q} 67 \mathrm{~L}}$ was stronger when compared with the interaction between $\mathrm{FgVps} 39$ and $\mathrm{FgRab} 7^{\mathrm{T} 22 \mathrm{~N}}$ in the $\mathrm{Y} 2 \mathrm{H}$ assay (Fig. 8A). This result was further confirmed by GST pull-down assay using the GST-FgRab7 ${ }^{\mathrm{Q} 67 \mathrm{~L}} / \mathrm{GST}$ $\mathrm{FgRab}^{\mathrm{T} 22 \mathrm{~N}}$ and His-FgVps39 fusion proteins. FgVps39 exhibited higher binding affinity to the GTP-associated version of FgRab7 than to the GDP-associated version of FgRab7 under the same conditions (Fig. 8B and C). These results suggest that FgVps39 binds the GTP-associated version of FgRab7 in F. graminearum.

\section{FgVps39 and FgSso1 are associated} with pigmentation in $F$. graminearum.

In order to understand whether the FgVam7-FgVps39-FgSso 1 complex is involved in pigmentation metabolism, wild-type PH-1 and the $\Delta F g v a m 7, \Delta F g v p s 39$, and $\Delta F g$ ssol mutants were cultured in potato dextrose broth medium for 3 days. The results showed that aurofusarin in the $\Delta F g v p s 39$ and $\Delta F g s s o l$ mutants was dramatically decreased and yellow pigment was significantly increased when compared with those in the wild type, whereas the $\Delta$ Fgvam 7 mutant displayed no significant
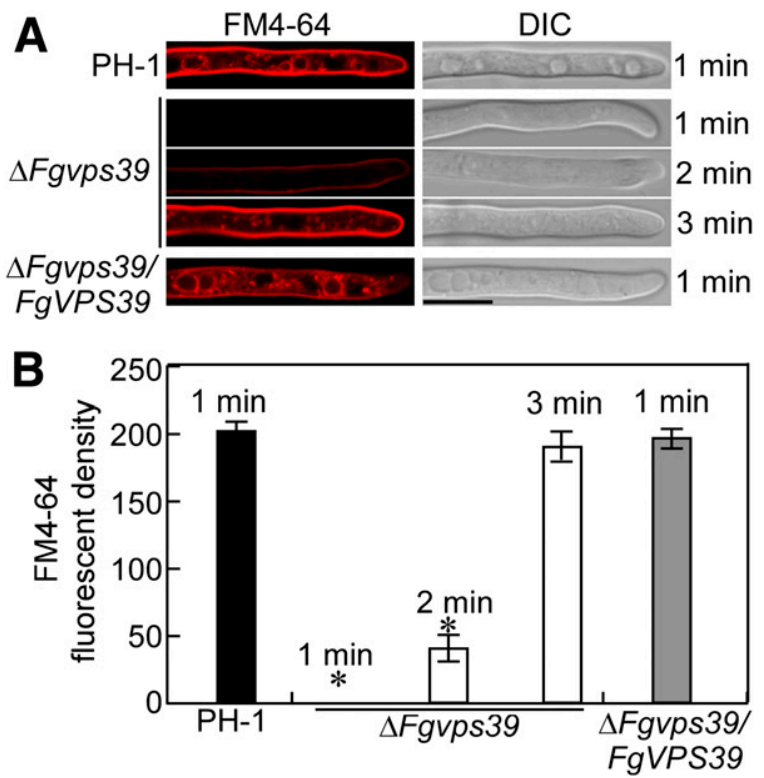

Fig. 7. FgVps39 plays a role in endocytosis of Fusarium graminearum. A, Hyphae of the indicated stains were cultured for $12 \mathrm{~h}$ on complete-medium-overlaid microscope slides before staining with FM4-64 dye and were examined by differential interference contrast (DIC) or epifluorescence microscopy. Bar $=10 \mu \mathrm{m}$. B, Statistical analysis of the relative fluorescent density. Error bars represent the standard deviations and asterisks indicate a statistically significant difference $(P<0.01)$.

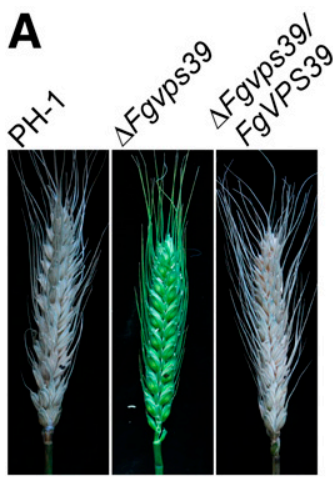

D

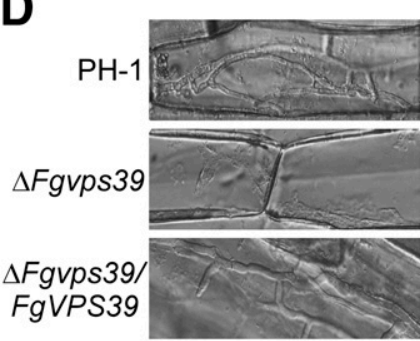

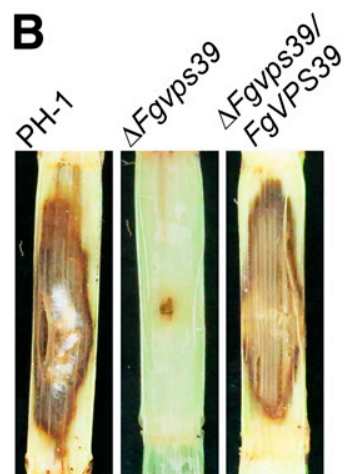
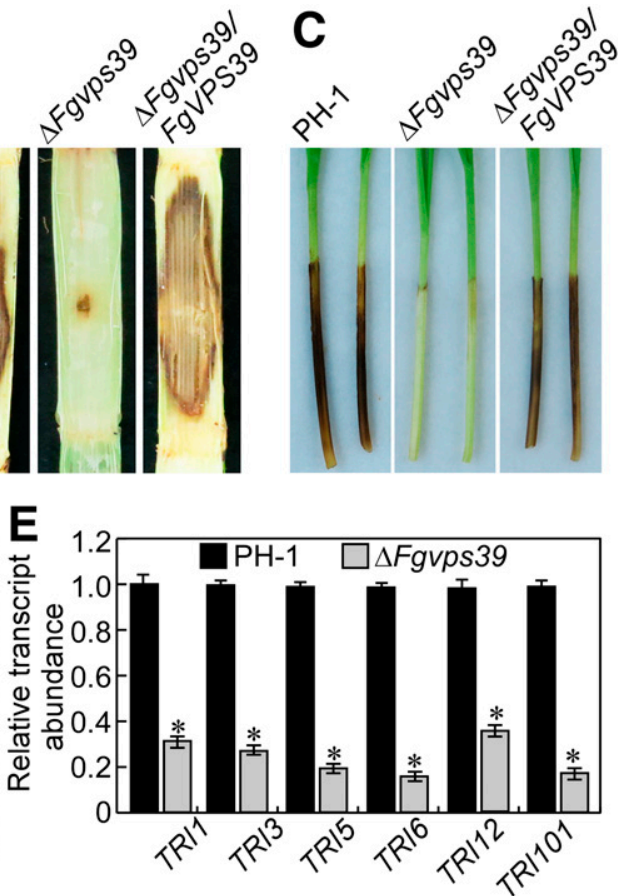

Fig. 6. Impact of FgVPS39 deletion on virulence and the expression of deoxynivalenol biosynthesis-related genes in Fusarium graminearum. A, Infection assays with conidia of PH-1, $\Delta$ Fgvps39, and $\Delta F$ gvps39/FgVPS39 were performed by drop inoculating onto flowering wheat heads. Photographs were taken at 14 days postinoculation (dpi). B, Conidia of the indicated strains were inoculated into corn stalks and examined at 7 dpi. C, Conidial suspensions harvested from the indicated strains were inoculated onto the wheat coleoptiles, as described (Zhang et al. 2012), and photographed at 10 dpi. D, Examination the infectious hyphae of the indicated strains on wheat coleoptiles at 2 dpi. E, Transcription levels of TRI1, TRI3, TRI5, TRI6, TRI12, and TRI101 were measured by quantitative real-time polymerase chain reaction. Asterisks indicate a statistically significant difference between wild-type PH- 1 and the $\Delta F g v p s 39$ mutant $(P<0.01)$. 
changes (Fig. 9A). We further detected the expression levels of seven aurofusarin biosynthetic-related genes. qRT-PCR assays showed that $A U R F, A U R J, A U R O, A U R R 1, A U R R 2$, and GIP1 were significantly downregulated in the $\Delta$ Fgvps39 and $\Delta F g$ ssol mutants (Fig. 9B). These results indicate that FgVps39 and FgSso1 are related to pigmentation by regulating aurofusarin synthesis in $F$. graminearum.

\section{The FgVps39-FgVam7-FgSso1 complex is important for autophagy in $F$. graminearum.}

Autophagy is a well-conserved process in which cytosol and organelles can be degraded and recycled in all eukaryotic cells (Liu et al. 2015). Because the $\Delta$ Fgvps39 mutant has defects in vacuole fusion, we supposed that it may also have a defect in autophagy. To test the possibility, we examined vacuoles of hyphal cells by starvation induction assay. We first examined the autophagic bodies under transmission electron microscopy. After being cultured in liquid MM-N medium (minimal medium without $\mathrm{NaNO}_{3}$ ) with $2 \mathrm{mM}$ phenylmethpanesulfonyl fluoride (PMSF) (proteinase B inhibitor: avoiding autophagic body degradation) for $4 \mathrm{~h}$, no autophagic bodies were detected in the vacuole of the $\Delta F g v p s 39$ mutant, whereas many autophagic bodies were found in the wildtype PH-1 (Fig. 10A).

The autophagic process could be observed by monitoring the vacuolar delivery and the subsequent breakdown of GFP-Atg8 by vacuolar hydrolases (Cheong and Klionsky 2008; Liu et al. 2015). The intact GFP moiety cleaved from GFP-Atg8 is relatively resistant to vacuolar proteolysis and accumulates in the vacuole when autophagic flux is normal (Cheong and Klionsky 2008). After $4 \mathrm{~h}$ of starvation induction, strong GFP-Atg8 signals were observed in the vacuole of wild-type PH-1. However, GFP-Atg8 exists in punctuate structures and surrounding the fragmented vacuoles in the $\Delta F g v p s 39$ mutant under the same conditions (Fig. 10B).

We also performed a GFP-FgAtg8 proteolysis assay for a more systematic evaluation. Under normal conditions, a distinct GFP-FgAtg8 band $(40 \mathrm{kDa})$ and a slightly weak GFP band (26 kDa) were detected in both the wild type and the $\Delta F g v p s 39$ mutant using an anti-GFP antibody (Fig. 10C). When the hyphae were shifted to MM-N conditions, a relatively weak full-length GFP-FgAtg8 band and a very clear GFP band were detected in the wild type. However, a very clear full-length band and a very weak GFP band were detected in the $\Delta F g v p s 39$ mutant (Fig. 10C). Similar results were observed in the $\Delta$ Fgvam 7 mutant (Fig. $10 \mathrm{~A}$ to $\mathrm{C}$ ). Interestingly, the $\Delta F$ gssol mutant showed no defect in vacuole fusion but a decrease in the number of autophagic bodies and a defect in GFP-FgAtg8 proteolysis were observed along with proper localization under inductive conditions (Fig. 10A to D).
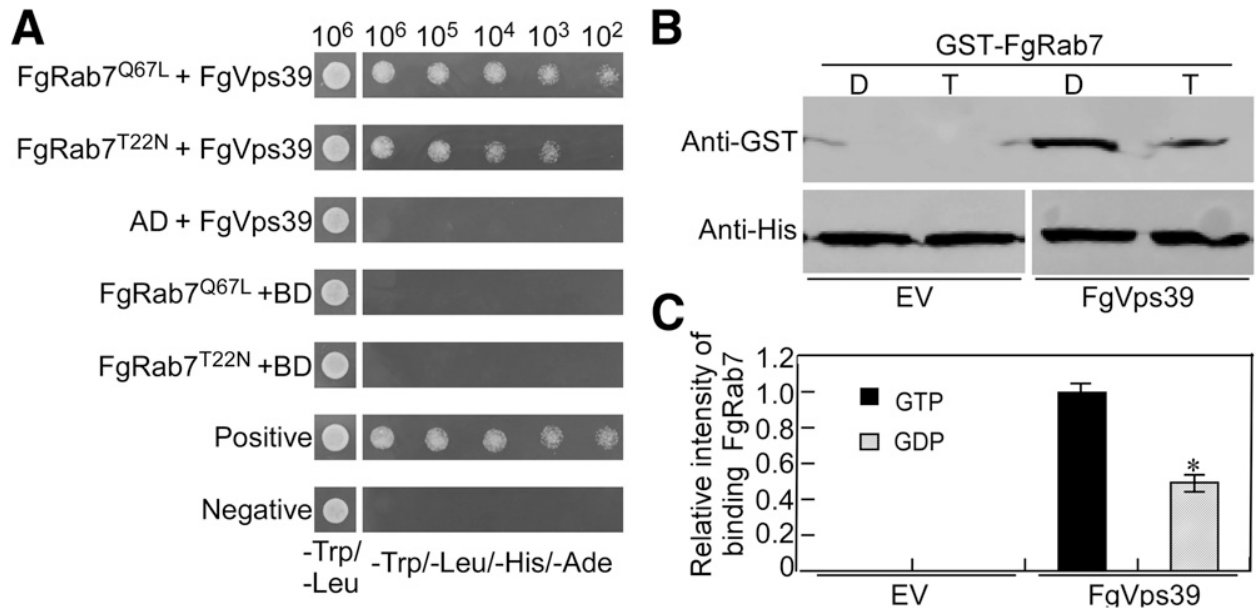

Fig. 8. Assays for the ability of FgVps39-binding FgRab7 $7^{\mathrm{Q} 67 \mathrm{~L}}$ and $\mathrm{FgRab} 7^{\mathrm{T} 22 \mathrm{~N}}$. A, Yeast two-hybrid assays for the interaction of FgVps39 between FgRab7 $^{\mathrm{Q} 67 \mathrm{~L}}$ and FgRab7 ${ }^{\mathrm{T} 22 \mathrm{~N}}$. pGBKT7-53/pGADT7-T and pGBKT7-Lam/pGADT7-T were used as positive and negative controls, respectively. B, FgVps39 directly binds FgRab7 (activated or inactivated). Recombinant GST-FgRab7 (activated or inactivated) to glutathione Sepharose beads decorated with HisFgVps39; the pET32a was used as control. GST, glutathione-S-transferase; His, histone; EV, empty vector. C, Quantified band intensities using the Odyssey infrared imaging system. Error bars represent the standard deviations and asterisks indicate a statistically significant difference $(P<0.01)$.
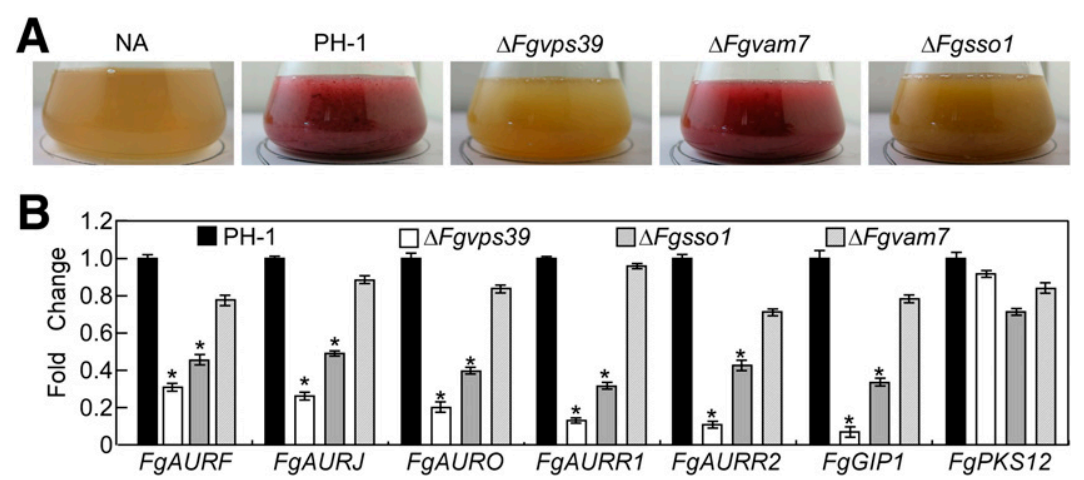

Fig. 9. Assays for the defects of the $\Delta F g v p s 39, \Delta F g v a m 7$, and $\Delta F g s s o 1$ mutants on pigment formation. A, Indicated strains were inoculated in liquid potato dextrose broth medium for 3 days and photographed. B, Relative expression levels of aurofusarin biosynthesis-related genes $A U R F, A U R J, A U R O, A U R R I$, AURR2, GIP1, and PKS12 in the $\Delta F$ gvps 39, $\Delta F g s s o 1$, and $\Delta F g v a m 7$ mutants. Total RNA was extracted from mycelia cultured in complete medium for 2 days. Error bars represent the standard deviations and asterisks indicate a statistically significant difference $(P<0.01)$. 
Effects of deleting FgVps39 on sensitivity of $F$. graminearum to fungicides and various environmental stresses.

Because the $\Delta$ Fgvps 39 mutant was defective in vacuolar morphology and delayed endocytosis, we examined the response of the mutant to various stresses such as fungicides and oxidative, osmotic, and salt stress. First, the fungicides azoxystrobin, carbendazim, and tebuconazole were added to CM plates at various concentrations. The inhibition rate of the $\Delta$ Fgvps 39 mutant decreased significantly on plates with azoxystrobin at 1 and $5 \mathrm{ppm}$ (Table 2). In contrast, the inhibition rate increased dramatically in response to tebuconazole at 0.03 and $0.12 \mathrm{ppm}$ (Table 2). Meanwhile, the inhibition rate of the $\Delta$ Fgvps39 mutant increased on plates with carbendazim at 0.06 ppm but decreased at $0.12 \mathrm{ppm}$ (Table 2). Additionally, the $\Delta$ Fgvps39 mutation increased sensitivity to salt $(1 \mathrm{M} \mathrm{NaCl}$ and
$1 \mathrm{M} \mathrm{KCl}$ ) and osmotic stress (1 M sorbitol) but decreased sensitivity to $\mathrm{H}_{2} \mathrm{O}_{2}$ oxidative stress (Table 2).

\section{DISCUSSION}

The transport of proteins and lipids mediated by vesicle trafficking is required for normal development of the cells (Bröcker et al. 2010). Our previous study reported that the SNARE protein FgVam7 mediates vesicle trafficking and is important for the development, DON production, and virulence of $F$. graminearum (Zhang et al. 2016). However, the detailed mechanism of vesicle trafficking regulated by FgVam7 is not clarified. Here, we identified a series of binding proteins of FgVam7 using an affinity purification approach, and found that the candidates $\mathrm{FgVps} 39$ and $\mathrm{FgSso1}$ have a role related to the vesicle trafficking pathway. FgSso1 is a SNARE protein that
A

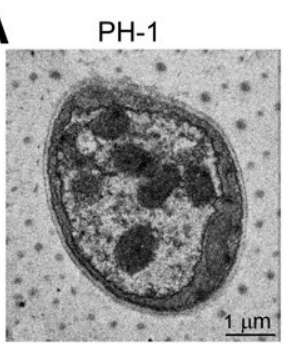

B

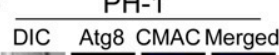

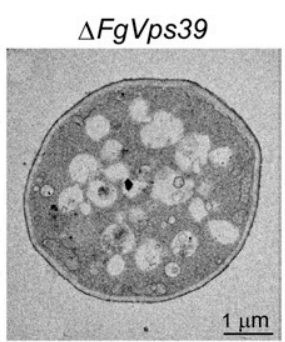

$\triangle F g \vee p s 39$

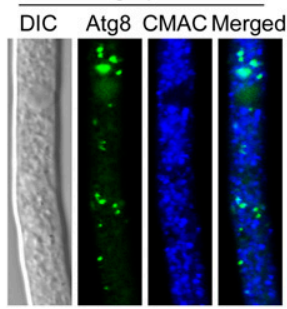

C

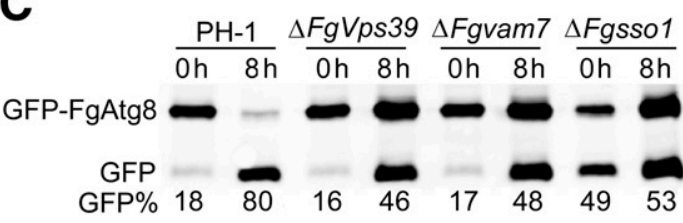

$\Delta$ Fgvam7

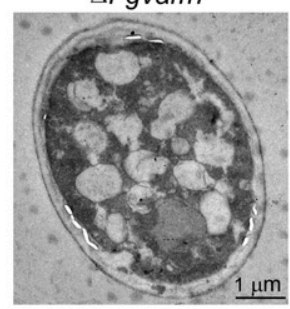

$\triangle$ Fgvam7

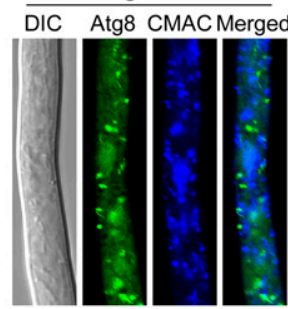

D

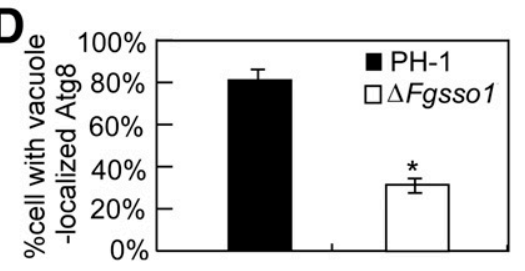

Fig. 10. Assays for the defects of the $\Delta F g v p s 39, \Delta F g v a m 7$, and $\Delta F g s s o 1$ mutants in autophagy. A, Mycelia of indicated strains induced in liquid MM-N medium (minimal medium without $\mathrm{NaNO}_{3}$ ) with $4 \mathrm{mM}$ phenylmethpanesulfonyl fluoride for $4 \mathrm{~h}$. Organelles and autophagic bodies were observed by transmission electron microscope. B, Green fluorescent protein (GFP)-FgAtg8 and 7-amino-4-chloromethylcoumarin (CMAC) staining observation of the indicated strains under starvation conditions by DIC or fluorescence microscopy. Bar $=10 \mu \mathrm{m}$. C, GFP-FgAtg8 proteolysis assays of PH-1 and the $\Delta F g v p s 39, \Delta F g s s o 1$, and $\Delta F g v a m 7$ mutants. Protein was extracted from mycelia cultured in liquid complete medium for $16 \mathrm{~h}$ or induced in MM-N for $8 \mathrm{~h}$, then analyzed by Western blot using antiGFP. D, Statistical analysis of 100 cells from $\Delta$ Fgssol (B) for autophagy, as above. DIC, differential interference contrast microscopy.

Table 2. Effects of FgVPS39 deletion on sensitivity of Fusarium graminearum to fungicides and various environmental stresses ${ }^{\text {a }}$

\begin{tabular}{|c|c|c|c|c|c|c|c|c|c|c|}
\hline \multirow[b]{2}{*}{ Strain } & \multicolumn{2}{|c|}{ Azoxystrobin ${ }^{b}$} & \multicolumn{2}{|c|}{ Carbendazim $^{c}$} & \multicolumn{2}{|c|}{ Tebuconazole $^{d}$} & \multicolumn{4}{|c|}{ Inhibition rate $(\%)^{\mathrm{e}}$} \\
\hline & 1 ppm & 5 ppm & 0.06 ppm & $0.12 \mathrm{ppm}$ & 0.03 ppm & $0.12 \mathrm{ppm}$ & $1 \mathrm{M} \mathrm{NaCl}$ & $1 \mathrm{M} \mathrm{KCl}$ & 1 M Sorb & $5 \mathrm{mM} \mathrm{H}_{2} \mathrm{O}_{2}$ \\
\hline PH-1 & $60.9 \pm 5.3$ & $75.3 \pm 3.4$ & $56.4 \pm 5.1$ & $90.9 \pm 4.2$ & $56.3 \pm 3.2$ & $81.8 \pm 1.3$ & $40.8 \pm 1.3$ & $32.9 \pm 0.0$ & $18.7 \pm 1.3$ & $18.8 \pm 2.3$ \\
\hline$\Delta F g v p s 39$ & $32.6 \pm 3.7^{*}$ & $46.7 \pm 4.2 *$ & $75.2 \pm 3.0^{*}$ & $84.6 \pm 1.2^{*}$ & $85.2 \pm 7.1 *$ & $91.1 \pm 4.2 *$ & $60.6 \pm 1.9^{*}$ & $38.8 \pm 2.3^{*}$ & $26.5 \pm 2.3^{*}$ & $2.9 \pm 0.0 *$ \\
\hline$\Delta F g v p s 39 / F g V P S 39$ & $61.2 \pm 2.5$ & $73.9 \pm 8.1$ & $54.8 \pm 6.2$ & $91.4 \pm 3.8$ & $54.7 \pm 5.3$ & $80.8 \pm 5.2$ & $38.8 \pm 2.3$ & $34.4 \pm 1.4$ & $19.6 \pm 1.2$ & $20.7 \pm 1.4$ \\
\hline
\end{tabular}


localized in the vacuole and endosomes, and is important for the development and virulence in $F$. graminearum (Hong et al. 2010), whereas $\mathrm{FgVps} 39$ is the homolog of yeast Vps39, a member of HOPS complexes that are involved in endosome to vacuole transportation (Balderhaar and Ungermann 2013). The mutant with a deletion of $F g V P S 39$ showed defects in growth, asexual or sexual development, DON production, and virulence that are consistent with the $\Delta$ Fgvam 7 mutant. Furthermore, FgVam7, $\mathrm{FgVps} 39$, and FgSsol were confirmed to interact with each other; therefore, we conclude that FaVam7 likely binds FgVps39 and FgSsol to participate in vesicle trafficking, thereby controlling the development and virulence in $F$. graminearum.

The HOPS complex is one of the most important trafficking systems, and its members are highly conserved in eukaryotic cells (Balderhaar and Ungermann 2013). In S. cerevisiae, Vps39, a GTP exchange factor (Wurmser et al. 2000), is a member of the HOPS complex (Starai et al. 2008) and is involved in vesicle trafficking from endosome to vacuole (Balderhaar and Ungermann 2013). In the filamentous fungus Aspergillus nidulans, disruption of a Vps39 homolog $a v a B$ resulted in fragmented vacuoles and a retarded growth phenotype under various growth conditions, implying its importance in maintaining vacuolar morphology and function (Oka et al. 2004). Consistent with these results, FgVps39 also plays important roles during the development and virulence mediated by vesicle trafficking. These results indicated that Vps39 proteins have conserved roles in different organisms.

A previous study showed that the SNARE protein MoSyn8 localized in endosomes, mediated vesicle trafficking, and controlled development and virulence in M. oryzae (Qi et al. 2016). In plants, vesicle trafficking mediates the interaction between TaSYP132 (a plant defense-related gene) and TaNPSN11 (a novel plant SNARE gene) during plant immunity (Wang et al. 2014). These results indicated that the SNARE proteins were associated with vesicle trafficking in eukaryotic organisms. The endosome and vacuole are two components of vesicle trafficking (Shoji et al. 2006). In A. oryzae, the early endosome moves along the hyphal axis throughout the hyphal and the late endosome or prevacuolar compartments, adjacent the vacuole, and can be stained with FM4-64 but not with CMAC (Shoji et al. 2006). In our study, FgVps39 colocalized with the early or late endosomes and vacuole, indicating that FgVps39 is involved in vesicle trafficking from early or late endosomes to vacuoles. In addition, the movies showed high mobility of FgVps39-RFP in endosomes in a tubulin filament-dependent way. In general, the fusion of vacuoles and endosomes required a conserved machinery that consists of Rab GTPases and their interacting effector, and SNARE proteins (Bonifacino and Glick 2004). Our previous study showed that the SNARE protein FgVam7 localized to vacuoles (Zhang et al. 2016). Here, we found that FgVps39, an effector of Rab7, localized in early or late endosomes and vacuoles. We speculate that FgVam7 interacted with FgVps39, likely in the fusion of late endosomes and vacuoles. Furthermore, we found that FgVam7 interacts with FgVps39 and FgSso1, suggesting the existence of an FgVps39-FgVam7-FgSso1 complex module that mediates vesicle trafficking. This complex is different from the reported retromer complex (Vps35, Vps5, Vps17, Vps26, and Vps29) and Rab GFPase complex, which mainly mediate the endosome-to-trans-Golgi network or play conserved roles as regulators (membrane-associated molecular switches) of intracellular vesicle trafficking such as extracellular protein secretion pathways (Stenmark 2009; Zheng et al. 2016). In addition, the $\Delta$ Fgvam $7, \Delta F g v p s 39$, and $\Delta F g$ ssol mutants shared similar phenotypes in vegetative growth and plant infection. This finding will further deepen our understanding of the $\mathrm{FgVps} 39-\mathrm{FgVam} 7-\mathrm{FgSso} 1$ complex in fungal pathogenicity, which has not been reported in pathogenic fungi.
In filamentous fungi, the development of hyphae requires many matrix components that are delivered by vesicle trafficking (Gupta and Brent Heath 2002). In S. cerevisiae, Vps39 promotes membrane fusion events at the late endosome to vacuole stage and is essential for membrane and protein trafficking (Balderhaar and Ungermann 2013). The reduced growth rate of the Fgvps39 mutant indicated that FgVps39 may mediate vesicle trafficking and transportation of matrix components. Several studies have shown that genes important for membrane fusion of the endomembrane system often play critical roles in the development and pathogenesis of plant-pathogenic fungi (Dou et al. 2011; Qi et al. 2016; Zheng et al. 2015). For example, deletion of the early endosomal marker FgRab51 and late endosomal marker FgRab7 in F. graminearum results in defects in development and plant infection (Zheng et al. 2015). At the same time, the abnormal motility of early endosomes of the smut fungus Ustilago maydis resulted in effector production and secretion defects during plant infection (Bielska et al. 2014). Our findings provide evidence that $\mathrm{FgVps} 39$ is involved in vesicle trafficking from the endosomes to the vacuole in $F$. graminearum, which is important for proper development and infection in this fungus.

The fungus produces secondary metabolites in development processes such as DON and zearalenon that are harmful to the health of human and animals (Chanda et al. 2009). A previous study showed that the synthesis and export of aflatoxin is mediated by vesicles and aflatoxigenic vesicles (aflatoxisomes), which are enhanced under aflatoxin-inducing growth conditions in A. parasiticus (Chanda et al. 2009). In our study, the lower level of DON in the $\Delta F g v p s 39$ mutant indicated that FgVps39 likely has a role in the transportation and synthesis of the toxin. In addition, DON is known as one of the important factors for infection of $F$. graminearum (Yu et al. 2014), and the lower levels of DON produced by the $\Delta F g v p s 39$ mutant probably contribute to its defect in virulence. Previous studies reported that the DON biosynthesis-related proteins contribute to DON production in $F$. graminearum (Menke et al. 2012, 2013) and the retromer complex (Vps35, Vps5, Vps17, Vps26, and $\mathrm{Vps} 29$ ) was reported to regulate the localization of Tri proteins in $F$. graminearum and mediate their recycling before translocation to the vacuole, thus controlling DON production. Our data showed that the expression levels of six DON biosynthesis-related genes were decreased in the $\Delta$ Fgvps 39 mutant. Therefore, we speculate that the vesicle trafficking might have a role in regulating the expression of DON biosynthesis-related genes to control DON production.

The Rab7 proteins in F. graminearum and M. oryzae localize to the late endosomes, which are similar to their orthologs in S. cerevisiae and Arabidopsis (Cui et al. 2014; Limpens et al. 2009; Liu et al. 2015; Nahm et al. 2003; Zheng et al. 2015; Zheng et al. 2016). The development of early endosomes to late endosomes in yeast and animal cells requires the conversion of Rab5 to Rab7. The Mon1-Ccz1 complex, a late-endosome GEF, could be recruited to the membrane by the activated Rab5 protein and binds to Rab7. In addition, the Mon1-Ccz1 complex also affects Rab7 activation, and acts as an important link between Rab5 and Rab7 (Kinchen and Ravichandran 2010; Nordmann et al. 2010; Rink et al. 2005). Therefore, wild-type Rab7 should contain GDP- and GTP-bound forms. Our previous study showed that FgMon1, as an FgRab7 GEF, interacts with the GTP-associated version of FgRab7 ${ }^{\mathrm{Q} 67 \mathrm{~L}}$ and the GDPassociated version of FgRab7 $7^{\mathrm{T} 22 \mathrm{~N}}$ in $\mathrm{Y} 2 \mathrm{H}$ and in vitro GST pulldown assays ( $\mathrm{Li}$ et al. 2015). Our results showed that FgVps39 competitively bound the GTP-associated version of FgRab7, indicating that $\mathrm{FgVps} 39$ plays roles downstream of the active GTP-bound form of Rab7 to take part in vacuole membrane fusion of $F$. graminearum. 
A previous study confirmed that different transport pathways converge upon the vacuole and that the CVT process overlaps with macroautophagy and is a conserved mechanism in eukaryotic cells (Chen et al. 2014; Nakatogawa et al. 2009). The autophagy pathway that delivers organelles and cytoplasmic content to the vacuole has been shown to be essential for fungal development and virulence (Bartoszewska and Kiel 2011; Gao et al. 2013; Liu et al. 2012). Fusion of the autophagosome with the vacuole or lysosome is the key step for the ultimate degradation of cytosolic materials by autophagy. GFP-Atg8 technology was established to label autophagosomes and autophagic vacuoles in filamentous fungi (Mizushima et al. 2004). In addition, the autophagy process also includes membrane trafficking and fusion events. Proteins involved in vesicle trafficking such as SNARE and Rab GTPases have been reported to be essential in autophagy (Liu et al. 2015; Zheng et al. 2015). Because the $\Delta$ Fgvps39 mutant was defective in endocytosis and vacuole fusion, we speculated that the $\Delta F g v p s 39$ mutant deficient in fusion of autophagosomes and vacuoles may also be due to fragmented vacuoles in the mutant. Previous studies reported that no autophagic bodies were examined in vacuoles of the $\Delta$ Moatgl mutant (Liu et al. 2007). The movement of GFPAtg8 to the vacuole was prevented in the $\Delta$ Moatgl mutant under induced conditions (Liu et al. 2007). However, GFPMoAtg8 showed a punctate distribution and surrounded the fragmented vacuoles in the $\Delta F g v p s 39$ mutant. Therefore, we speculate that MoAtg1 and FgVps39 are likely involved in different steps of the autophagic processes. The $\Delta F g v p s 39$ mutant was defective in fusion of autophagosomes. However, the exact nature of GFP-Atg8 proteolysis during autophagy in fungi remains unclear.

Taken together, we identified the FgVam7 binding proteins $\mathrm{FgVps} 39$ and FgSso1, and demonstrated that FgVam7, FgVps39, and FgSsol interact with each other as a complex and mediate vesicle trafficking for hyphal growth, sexual reproduction, pathogenesis, vacuole fusion, endocytosis, and autophagy (Fig. 11). These interactions are conserved but different from those in yeast and animals, in which Vps39 functions as a scaffold protein and interacts with Vam7 and Sso1, although there is no interaction between Vam7 and Sso1 (Aalto et al. 1993; Jahn and Scheller 2006). Although the model of action remains poorly understood, our study provides evidence to clarify the dynamic trafficking process mediated by the FgVps39-FgVam7-FgSso 1 complex. Further studies will be focused on the mechanisms of toxin biosynthesis and transportation mediated by vesicle trafficking in $F$. graminearum.

\section{MATERIALS AND METHODS}

Strains of $\boldsymbol{F}$. graminearum and growth conditions.

The F. graminearum wild-type strain $\mathrm{PH}-1$ (Cuomo et al. 2007), all mutants, and the complemented transformants were cultured at $25^{\circ} \mathrm{C}$ in V8 juice agar medium. For sexual reproduction, $\mathrm{PH}-1$ and the derived mutants were inoculated on carrot agar plates, as described (Klittich and Leslie 1988). Cultures for genomic DNA and RNA isolation, conidiation in CMC medium, and growth assays on CM, MM, and 5× YEG medium were performed as previously described (Zhang et al. 2016). Protoplast preparation and transformation followed previous procedures (Zhou et al. 2011). Hygromycin B (Roche) and neomycin (Sangon Biotech) were used to screen the transformants at a final concentration of 300 and $400 \mu \mathrm{g} / \mathrm{ml}$, respectively.

\section{Targeted gene deletion and complementation.}

The $\Delta$ Fgvps 39 mutant was generated with the split-marker approach, as previously described (Zhang et al. 2016). The complemented construct was prepared by cloning the entire FgVPS39 gene into pYF11 using the yeast gap repair approach (Zhou et al. 2011). The neomycin-resistant transformants were screened by PCR and GFP signal examination. The primers used in this section are listed in Supplementary Table S2.

\section{Plant infection and DON production assays.}

For infection assays, conidia preparation and plant inoculation were performed as described (Zhang et al. 2016). For result analysis, at least 20 infected wheat heads were determined for each strain. For DON production analysis, five mycelia dishes of each strain were inoculated with $50 \mathrm{~g}$ of healthy and autoclaved wheat kernels (Yun et al. 2014). The wheat kernels were inoculated at $25^{\circ} \mathrm{C}$ for 20 days. DON and fungal ergosterol were extracted as described (Mirocha et al. 1998). PuriTox SR DON colummTC-T200 (Trilogy) was used to purify the DON. The experiments were repeated three times, with three replicates each time.

\section{qRT-PCR.}

Total RNA samples were extracted from vegetative hyphae of each sample using the Nucleo Spin RNAII RNA extraction kit (Invitrogen), and used for cDNA synthesis with M-MLV reverse transcription (Vazyme Biotech) following the instructions. The RT2 PCR Real-Time SYBR Green/ROX PCR master

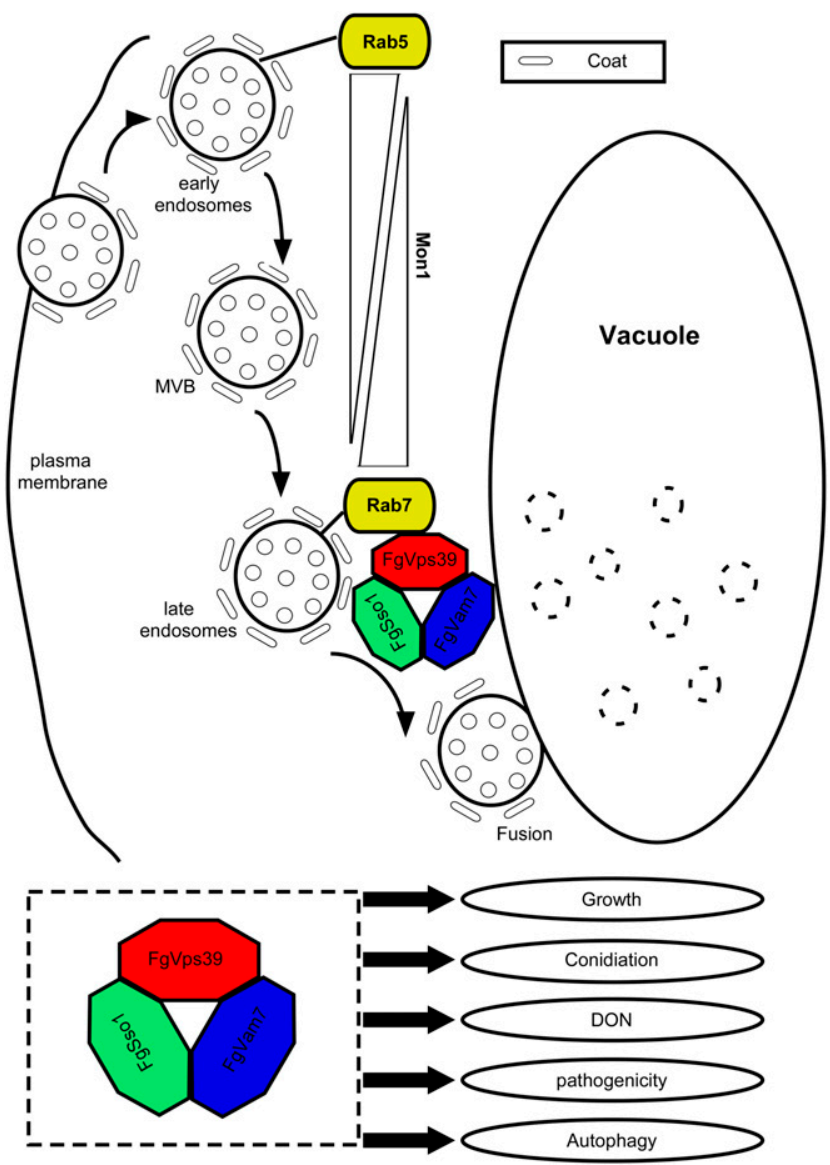

Fig. 11. Proposed model for the involvement of the FgVps39-FgVam7-FgSso1 complex in vesicle trafficking pathway in Fusarium graminearum. The dashed box indicates that $\mathrm{FgVam} 7, \mathrm{FgVps} 39$, and FgSso1 interact with each other as a complex. The function of FgVps39-FgVam7-FgSso1 is involved in vesicle trafficking at endosomes to vacuole. FgVps39 is colocalized with the microtubule and moves through a microtubule network. Deletion of any member of the complex affects normal development, virulence, and deoxynivalenol (DON) production of $F$. graminearum. MVB, multivesicular body. 
mix (TaKaRa) was used for qRT-PCR analysis. To compare the relative abundance of target gene transcripts, the average threshold cycle $(\mathrm{Ct})$ was normalized to that of Actin ( $\beta$-tubulin) for each of the treated samples as $2^{-\Delta \mathrm{Ct}}$, where $-\Delta \mathrm{Ct}=\left(\mathrm{Ct}_{\text {target gene }}-\mathrm{Ct}_{\text {actin }}\right)$. Relative transcript abundance during development and infectious growth compared with growth in liquid $\mathrm{CM}$ were calculated as $2^{-\Delta \Delta \mathrm{Ct}}$, where $-\Delta \Delta \mathrm{Ct}=\left(\mathrm{Ct}_{\text {target gene }}-\mathrm{Ct}_{\mathrm{actin}}\right)_{\text {test condition }}-\left(\mathrm{Ct}_{\mathrm{WT}}-\right.$ $\left.\mathrm{Ct}_{\mathrm{actin}}\right)_{\mathrm{CM}}$. The qRT-PCR assay was repeated three times with three biological replicates.

\section{Light microscopy observation.}

To examine endocytosis, hyphae of the wild-type $\mathrm{PH}-1$ and mutant were prepared in liquid CM medium for $12 \mathrm{~h}$ and stained by FM4-64, as described (Fischer-Parton et al. 2000). Photographs were taken under the Zeiss LSM 710 confocal microscope using 63X/1.2 NA C-Apochromat oil immersion objectives, and Image-pro Plus was used to analyze the relative fluorescent (Media Cybernetics, Inc.).

\section{Y2H and in vitro pull-down assays.}

For $\mathrm{Y} 2 \mathrm{H}$, bait constructs were generated by cloning FgVAM7 and FgSSO1 full-length cDNA sequences into the GAL4 binding-domain vector pGBKT7 (Clontech). FgVPS39 and $\mathrm{FgSSO} 1$ full-length cDNA sequence was cloned into the GAL4 activation-domain vector pGADT7 (Clontech) as the prey constructs. The pairs of the plasmids were cotransformed into the yeast strain AH109 following the transformation protocol (Schiestl and Gietz 1989). In addition, Plasmids pGBKT753/pGADT7 and pGBKT7-Lam/pGADT7 were used as positive and negative controls, respectively. Transformants were grown on SD-Leu-Trp for 3 days, and assayed for growth on SD-Trp-Leu-His containing $3 \mathrm{mM} 3 \mathrm{AT}$ to assess binding activity (Cohen and Schuldiner 2011) or SD-Trp-Leu-His-Ade for 3 days. For in vitro GST pull-down assay, fused GST tag constructs were generated by cloning $\mathrm{FgVPS} 39$ and $\mathrm{FgSSOl}$ full-length cDNA into vector pGEX-4T-2. Fused His tag constructs were generated by cloning FgVPS39 and FgVAM7 fulllength cDNA into vector pET-32a. The fused GST tag and His tag expressed constructs separately transformed into the $E$. coli BL21-CodonPlus (DE3) cells (Stratagene). Protein expression was induced for $8 \mathrm{~h}$ at $25^{\circ} \mathrm{C}$ after the addition of isopropy- $\beta$-Dthiogalactoside to a final concentration of $0.1 \mathrm{mM}$. Cells were collected by centrifugation and washed. To extract proteins, dells were lysed in lysis buffer $(0.5 \mathrm{mM}$ EDTA, $1 \%$ Triton, $20 \mathrm{mM}$ Tris- $\mathrm{HCl}, 0.15 \mathrm{M} \mathrm{NaCl}, 1 \mathrm{mM}$ dithiothreitol, and protease inhibitors [1 $\mathrm{mM}$ PMSF]) with an ultrasonic break instrument. For protein purification, supernatants were separately mixed with GST beads and His beads for $2 \mathrm{~h}$, centrifuged at $500 \mathrm{rpm}$ for $2 \mathrm{~min}$ at $4^{\circ} \mathrm{C}$, washed with Tris- $\mathrm{NaCl}$ buffer (1 mM PMSF, $1 \%$ Triton, $50 \mathrm{mM}$ Tris- $\mathrm{HCl}$, and $100 \mathrm{mM} \mathrm{NaCl}$ ) and the proteins were eluted with $15 \mathrm{mM}$ glutathione. Proteins were also verified by sodium dodecyl sulfate polyacrylamide gel electrophoresis (SDS-PAGE) analysis and Western blotting with the anti-GST and anti-His antibodies (Abmart). For binding, the GST protein extracts were added to glutathioneSepharose resin washed with Tris- $\mathrm{NaCl}$ buffer. The protein His supernatants were added to resin and incubated $4 \mathrm{~h}$ with gentle rotation at $4{ }^{\circ} \mathrm{C}$, precipitated, and washed three times with Tris$\mathrm{NaCl}$ buffer. SDS-containing gel-loading buffer $(100 \mu \mathrm{l})$ was added to the resin before a brief boiling. Samples were analyzed by SDS-PAGE electrophoresis and Western blotting using anti-His and anti-GST antibodies. GST protein was used as a control.

\section{Protein extraction and Western blot assays.}

Each tested strain was cultured in $150 \mathrm{ml}$ of $\mathrm{CM}$ liquid medium at $25^{\circ} \mathrm{C}$ and $160 \mathrm{rpm}$ for $24 \mathrm{~h}$. Mycelia were harvested and ground in liquid nitrogen. Subsequently, approximately $200 \mathrm{mg}$ of mycelial powder was suspended in $1 \mathrm{ml}$ of extraction buffer (50 mM Tris- $\mathrm{HCl}$ [pH 7.5], $100 \mathrm{mM} \mathrm{NaCl}, 5 \mathrm{mM}$ EDTA, $1 \%$ Triton $\mathrm{X}-100$, and $2 \mathrm{mM}$ PMSF) with $10 \mu \mathrm{l}$ of protease inhibitor cocktail (Sigma-Aldrich). The following steps and Western blot analysis were performed as described (Yu et al. 2014). For GFP-FgAtg8 proteolysis assay, the FgATG8 gene was amplified from wild-type genomic DNA with primers and cloned into pYF11 plasmid with the yeast gap repair approach (Zhou et al. 2011). The resulting GFP-FgAtg8 vector was transformed into $\mathrm{PH}-1$ and mutants using polyethylene glycolmediated transformation (Zhou et al. 2011). Transformants expressing the GFP-FgAtg8 construct were selected to detect the GFP-FgAtg8 proteolysis by Western blot with anti-GFP antibody.

\section{Statistical analysis.}

Three results were used to calculate the mean and standard deviation. The significant difference between treatments was defined using SD and one-way analysis of variance (ANOVA) by SPSS 2.0. Significance between a couple of samples was evaluated by ANOVA, followed by an $F$ test at $P<0.01$.

\section{ACKNOWLEDGMENTS}

We thank J.-R. Xu of Purdue University for critical reviewing and revising the manuscript for us. This research was supported by the National Basic Research Program of China (grant number 2013CB127800), the Natural Science Foundation of China (grant number 31471736, HZ), and the National Key Research and Development Program of China (grant number 2016YFD0300706).

\section{LITERATURE CITED}

Aalto, M. K., Ronne, H., and Keränen, S. 1993. Yeast syntaxins Sso1p and Sso2p belong to a family of related membrane proteins that function in vesicular transport. EMBO J. 12:4095-4104.

Bai, G. H., Desjardins, A. E., and Plattner, R. D. 2002. Deoxynivalenolnonproducing Fusarium graminearum causes initial infection, but does not cause disease spread in wheat spikes. Mycopathologia 153: 91-98.

Balderhaar, H. J., and Ungermann, C. 2013. CORVET and HOPS tethering complexes - coordinators of endosome and lysosome fusion. J. Cell Sci. 126:1307-1316.

Bartoszewska, M., and Kiel, J. A. 2011. The role of macroautophagy in development of filamentous fungi. Antioxid. Redox Signal. 14: 2271-2287.

Bielska, E., Higuchi, Y., Schuster, M., Steinberg, N., Kilaru, S., Talbot, N. J., and Steinberg, G. 2014. Long-distance endosome trafficking drives fungal effector production during plant infection. Nat. Commun. 5:Article number 5097

Binda, M., Péli-Gulli, M. P., Bonfils, G., Panchaud, N., Urban, J., Sturgill, T. W., Loewith, R., and De Virgilio, C. 2009. The Vam6 GEF controls TORC1 by activating the EGO complex. Mol. Cell 35:563-573.

Bonifacino, J. S., and Glick, B. S. 2004. The mechanisms of vesicle budding and fusion. Cell 116:153-166.

Bröcker, C., Engelbrecht-Vandré, S., and Ungermann, C. 2010. Multisubunit tethering complexes and their role in membrane fusion. Curr. Biol. 20:R943-R952.

Burri, L., and Lithgow, T. 2004. A complete set of SNAREs in yeast. Traffic 5:45-52.

Burri, L., Varlamov, O., Doege, C. A., Hofmann, K., Beilharz, T., Rothman, J. E., Söllner, T. H., and Lithgow, T. 2003. A SNARE required for retrograde transport to the endoplasmic reticulum. Proc. Natl. Acad. Sci. U.S.A. 100:9873-9877.

Chanda, A., Roze, L. V., Kang, S., Artymovich, K. A., Hicks, G. R., Raikhel, N. V., Calvo, A. M., and Linz, J. E. 2009. A key role for vesicles in fungal secondary metabolism. Proc. Natl. Acad. Sci. U.S.A. 106:19533-19538.

Chen, Y., Zhou, F., Zou, S., Yu, S., Li, S., Li, D., Song, J., Li, H., He, Z., Hu, B., Björn, L. O., Lipatova, Z., Liang, Y., Xie, Z., and Segev, N. 2014. A Vps21 endocytic module regulates autophagy. Mol. Biol. Cell 25: 3166-3177 
Cheong, H., and Klionsky, D. J. 2008. Biochemical methods to monitor autophagy-related processes in yeast. Methods Enzymol. 451:1-26.

Cohen, Y., and Schuldiner, M. 2011. Advanced methods for highthroughput microscopy screening of genetically modified yeast libraries. Methods Mol. Biol. 781:127-159.

Cui, Y., Zhao, Q., Gao, C., Ding, Y., Zeng, Y., Ueda, T., Nakano, A., and Jiang, L. 2014. Activation of the Rab7 GTPase by the MON1-CCZ1 complex is essential for PVC-to-vacuole trafficking and plant growth in Arabidopsis. Plant Cell 26:2080-2097.

Cuomo, C. A., Güldener, U., Xu, J. R., Trail, F., Turgeon, B. G., Di Pietro, A., Walton, J. D., Ma, L. J., Baker, S. E., Rep, M., Adam, G., Antoniw, J., Baldwin, T., Calvo, S., Chang, Y. L., Decaprio, D., Gale, L. R., Gnerre, S., Goswami, R. S., Hammond-Kosack, K., Harris, L. J., Hilburn, K., Kennell, J. C., Kroken, S., Magnuson, J. K., Mannhaupt, G., Mauceli, E., Mewes, H. W., Mitterbauer, R., Muehlbauer, G., Münsterkötter, M., Nelson, D., O’Donnell, K., Ouellet, T., Qi, W., Quesneville, H., Roncero, M. I. G., Seong, K. Y., Tetko, I. V., Urban, M., Waalwijk, C., Ward, T. J., Yao, J., Birren, B. W., and Kistler, H. C. 2007. The Fusarium graminearum genome reveals a link between localized polymorphism and pathogen specialization. Science 317:1400-1402.

Desai, A., and Mitchison, T. J. 1997. Microtubule polymerization dynamics. Annu. Rev. Cell Dev. Biol. 13:83-117.

Desjardins, A. E., Proctor, R. H., Bai, G. H., McCormick, S. P., Shaner, G., Buechley, G., and Hohn, T. M. 1996. Reduced virulence of trichothecenenonproducing mutants of Gibberella zeae in wheat field tests. Mol. PlantMicrobe Interact. 9:775-781.

Dou, X., Wang, Q., Qi, Z., Song, W., Wang, W., Guo, M., Zhang, H., Zhang, Z., Wang, P., and Zheng, X. 2011. MoVam7, a conserved SNARE involved in vacuole assembly, is required for growth, endocytosis, ROS accumulation, and pathogenesis of Magnaporthe oryzae. PLoS One 6 : e16439.

Fasshauer, D., Sutton, R. B., Brunger, A. T., and Jahn, R. 1998. Conserved structural features of the synaptic fusion complex: SNARE proteins reclassified as Q- and R-SNAREs. Proc. Natl. Acad. Sci. U.S.A. 95 15781-15786.

Fischer-Parton, S., Parton, R. M., Hickey, P. C., Dijksterhuis, J., Atkinson, H. A., and Read, N. D. 2000. Confocal microscopy of FM4-64 as a too for analysing endocytosis and vesicle trafficking in living fungal hyphae. J. Microsc. 198:246-259.

Gao, H. M., Liu, X. G., Shi, H. B., Lu, J. P., Yang, J., Lin, F. C., and Liu, X. H. 2013. MoMon1 is required for vacuolar assembly, conidiogenesis and pathogenicity in the rice blast fungus Magnaporthe oryzae. Res. Microbiol. 164:300-309.

Goswami, R. S., and Kistler, H. C. 2004. Heading for disaster: Fusarium graminearum on cereal crops. Mol. Plant Pathol. 5:515-525.

Gupta, G. D., and Brent Heath, I. 2002. Predicting the distribution, conservation, and functions of SNAREs and related proteins in fungi. Fungal Genet. Biol. 36:1-21.

Hong, S. Y., So, J., Lee, J., Min, K., Son, H., Park, C., Yun, S. H., and Lee, Y. W. 2010. Functional analyses of two syntaxin-like SNARE genes, $G z S Y N 1$ and $G z S Y N 2$, in the ascomycete Gibberella zeae. Fungal Genet Biol. 47:364-372.

Jahn, R., Lang, T., and Südhof, T. C. 2003. Membrane fusion. Cell 112 519-533.

Jahn, R., and Scheller, R. H. 2006. SNAREs-Engines for membrane fusion. Nat. Rev. Mol. Cell Biol. 7:631-643.

Kerr, M. C., Lindsay, M. R., Luetterforst, R., Hamilton, N., Simpson, F., Parton, R. G., Gleeson, P. A., and Teasdale, R. D. 2006. Visualisation of macropinosome maturation by the recruitment of sorting nexins. J. Cell Sci. 119:3967-3980.

Kinchen, J. M., and Ravichandran, K. S. 2010. Identification of two evolutionarily conserved genes regulating processing of engulfed apoptotic cells. Nature 464:778-782.

Klittich, C., and Leslie, J. F. 1988. Nitrate reduction mutants of Fusarium moniliforme (Gibberella fujikuroi). Genetics 118:417-423.

Li, L., and Guan, K. L. 2009. Amino acid signaling to TOR activation: Vam6 functioning as a Gtr1 GEF. Mol. Cell 35:543-545.

Li, Y., Li, B., Liu, L., Chen, H., Zhang, H., Zheng, X., and Zhang, Z. 2015. FgMon1, a guanine nucleotide exchange factor of FgRab7, is important for vacuole fusion, autophagy and plant infection in Fusarium graminearum. Sci. Rep. 5:Article number 18101.

Limpens, E., Ivanov, S., van Esse, W., Voets, G., Fedorova, E., and Bisseling, T. 2009. Medicago N2-fixing symbiosomes acquire the endocytic identity marker Rab7 but delay the acquisition of vacuolar identity. Plant Cell 21:2811-2828.

Liu, X. H., Chen, S. M., Gao, H. M., Ning, G. A., Shi, H. B., Wang, Y., Dong, B., Qi, Y. Y., Zhang, D. M., Lu, G. D., Wang, Z. H., Zhou, J., and Lin, F. C. 2015. The small GTPase MoYpt7 is required for membrane fusion in autophagy and pathogenicity of Magnaporthe oryzae. Environ Microbiol. 17:4495-4510.

Liu, X. H., Gao, H. M., Xu, F., Lu, J. P., Devenish, R. J., and Lin, F. C. 2012 Autophagy vitalizes the pathogenicity of pathogenic fungi. Autophagy 8:1415-1425

Liu, X. H., Lu, J. P., Zhang, L., Dong, B., Min, H., and Lin, F. C. 2007. Involvement of a Magnaporthe grisea serine/threonine kinase gene, $\mathrm{MgATG} 1$, in appressorium turgor and pathogenesis. Eukaryot. Cell 6:997-1005.

Luo, Y., Zhang, H., Qi, L., Zhang, S., Zhou, X., Zhang, Y., and Xu, J. R. 2014. FgKin1 kinase localizes to the septal pore and plays a role in hyphal growth, ascospore germination, pathogenesis, and localization of Tub1 beta-tubulins in Fusarium graminearum. New Phytol. 204:943-954

McMullen, M., Jones, R., and Gallenberg, D. 1997. Scab of wheat and barley: A re-emerging disease of devastating impact. Plant Dis. 81:1340-1348.

Menke, J., Dong, Y., and Kistler, H. C. 2012. Fusarium graminearum Tri12p influences virulence to wheat and trichothecene accumulation. Mol. Plant-Microbe Interact. 25:1408-1418.

Menke, J., Weber, J., Broz, K., and Kistler, H. C. 2013. Cellular development associated with induced mycotoxin synthesis in the filamentous fungus Fusarium graminearum. PLoS One 8:e63077.

Mirocha, C. J., Kolaczkowski, E., Xie, W. P., Yu, H., and Jelen, H. 1998. Analysis of deoxynivalenol and its derivatives (batch and single kernel) using gas chromatography mass spectrometry. J. Agric. Food Chem. 46: 1414-1418.

Mizushima, N., Yamamoto, A., Matsui, M., Yoshimori, T., and Ohsumi, Y. 2004. In vivo analysis of autophagy in response to nutrient starvation using transgenic mice expressing a fluorescent autophagosome marker. Mol. Biol. Cell 15:1101-1111.

Moisoi, N., Erent, M., Whyte, S., Martin, S., and Bayley, P. M. 2002. Calmodulin-containing substructures of the centrosomal matrix released by microtubule perturbation. J. Cell Sci. 115:2367-2379.

Morelli, E., Ginefra, P., Mastrodonato, V., Beznoussenko, G. V., Rusten, T. E., Bilder, D., Stenmark, H., Mironov, A. A., and Vaccari, T. 2014 Multiple functions of the SNARE protein Snap29 in autophagy, endocytic, and exocytic trafficking during epithelial formation in Drosophila. Autophagy 10:2251-2268.

Nahm, M. Y., Kim, S. W., Yun, D., Lee, S. Y., Cho, M. J., and Bahk, J. D 2003. Molecular and biochemical analyses of OsRab7, a rice Rab7 homolog. Plant Cell Physiol. 44:1341-1349.

Nakamura, N., Hirata, A., Ohsumi, Y., and Wada, Y. 1997. Vam2/Vps41p and Vam6/Vps39p are components of a protein complex on the vacuolar membranes and involved in the vacuolar assembly in the yeast Saccharomyces cerevisiae. J. Biol. Chem. 272:11344-11349.

Nakatogawa, H., Suzuki, K., Kamada, Y., and Ohsumi, Y. 2009. Dynamics and diversity in autophagy mechanisms: Lessons from yeast. Nat. Rev. Mol. Cell Biol. 10:458-467.

Nordmann, M., Cabrera, M., Perz, A., Bröcker, C., Ostrowicz, C., Engelbrecht-Vandré, S., and Ungermann, C. 2010. The Mon1-Ccz1 complex is the GEF of the late endosomal Rab7 homolog Ypt7. Curr Biol. 20:1654-1659.

Oka, M., Maruyama, J., Arioka, M., Nakajima, H., and Kitamoto, K. 2004. Molecular cloning and functional characterization of avaB, a gene encoding Vam6p/Vps39p-like protein in Aspergillus nidulans. FEMS Microbiol. Lett. 232:113-121.

Peplowska, K., Markgraf, D. F., Ostrowicz, C. W., Bange, G., and Ungermann, C. 2007. The CORVET tethering complex interacts with the yeast Rab5 homolog Vps21 and is involved in endo-lysosomal biogenesis. Dev. Cell 12:739-750.

Proctor, R. H., Hohn, T. M., and McCormick, S. P. 1995. Reduced virulence of Gibberella zeae caused by disruption of a trichothecene toxin biosynthetic gene. Mol. Plant-Microbe Interact. 8:593-601.

Qi, Z., Liu, M., Dong, Y., Zhu, Q., Li, L., Li, B., Yang, J., Li, Y., Ru, Y., Zhang, H., Zheng, X., Wang, P., and Zhang, Z. 2016. The syntaxin protein (MoSyn8) mediates intracellular trafficking to regulate conidiogenesis and pathogenicity of rice blast fungus. New Phytol. 209:1655-1667.

Ramanujam, R., Calvert, M. E., Selvaraj, P., and Naqvi, N. I. 2013. The late endosomal HOPS complex anchors active G-protein signaling essentia for pathogenesis in Magnaporthe oryzae. PLoS Pathog. 9:e1003527.

Rink, J., Ghigo, E., Kalaidzidis, Y., and Zerial, M. 2005. Rab conversion as a mechanism of progression from early to late endosomes. Cell 122: 735-749.

Rothman, J. E. 1994. Mechanisms of intracellular protein transport. Nature 372:55-63.

Rothman, J. H., and Stevens, T. H. 1986. Protein sorting in yeast: Mutants defective in vacuole biogenesis mislocalize vacuolar proteins into the late secretory pathway. Cell 47:1041-1051. 
Schiestl, R. H., and Gietz, R. D. 1989. High efficiency transformation of intact yeast cells using single stranded nucleic acids as a carrier. Curr. Genet. 16:339-346.

Seals, D. F., Eitzen, G., Margolis, N., Wickner, W. T., and Price, A. 2000. A Ypt/Rab effector complex containing the Sec1 homolog Vps33p is required for homotypic vacuole fusion. Proc. Natl. Acad. Sci. U.S.A. 97: 9402-9407.

Seong, K. Y., Pasquali, M., Zhou, X., Song, J., Hilburn, K., McCormick, S., Dong, Y., Xu, J. R., and Kistler, H. C. 2009. Global gene regulation by Fusarium transcription factors Tri6 and Tri10 reveals adaptations for toxin biosynthesis. Mol. Microbiol. 72:354-367.

Shoji, J. Y., Arioka, M., and Kitamoto, K. 2006. Vacuolar membrane dynamics in the filamentous fungus Aspergillus oryzae. Eukaryot. Cell 5:411-421.

Song, W., Dou, X., Qi, Z., Wang, Q., Zhang, X., Zhang, H., Guo, M., Dong, S., Zhang, Z., Wang, P., and Zheng, X. 2010. R-SNARE homolog MoSec22 is required for conidiogenesis, cell wall integrity, and pathogenesis of Magnaporthe oryzae. PLoS One 5:e13193.

Starai, V. J., Hickey, C. M., and Wickner, W. 2008. HOPS proofreads the trans-SNARE complex for yeast vacuole fusion. Mol. Biol. Cell 19: 2500-2508.

Stenmark, H. 2009. Rab GTPases as coordinators of vesicle traffic. Nat. Rev. Mol. Cell Biol. 10:513-525.

Valbuena, N., Guan, K. L., and Moreno, S. 2012. The Vam6 and Gtr1-Gtr2 pathway activates TORC1 in response to amino acids in fission yeast. J. Cell Sci. 125:1920-1928.

van der Kant, R., Jonker, C. T., Wijdeven, R. H., Bakker, J., Janssen, L., Klumperman, J., and Neefjes, J. 2015. Characterization of the mammalian CORVET and HOPS complexes and their modular restructuring for endosome specificity. J. Biol. Chem. 290:30280-30290.

Vida, T. A., and Emr, S. D. 1995. A new vital stain for visualizing vacuolar membrane dynamics and endocytosis in yeast. J. Cell Biol. 128:779-792.

Wang, X., Wang, X., Deng, L., Chang, H., Dubcovsky, J., Feng, H., Han, Q., Huang, L., and Kang, Z. 2014. Wheat TaNPSN SNARE homologues are involved in vesicle-mediated resistance to stripe rust (Puccinia striiformis f. sp. tritici). J. Exp. Bot. 65:4807-4820.

Wartosch, L., Günesdogan, U., Graham, S. C., and Luzio, J. P. 2015. Recruitment of VPS33A to HOPS by VPS16 is required for lysosome fusion with endosomes and autophagosomes. Traffic 16:727-742.
Wurmser, A. E., Sato, T. K., and Emr, S. D. 2000. New component of the vacuolar class C-Vps complex couples nucleotide exchange on the Ypt7 GTPase to SNARE-dependent docking and fusion. J. Cell Biol. 151: 551-562.

Yang, X., Cui, H., Cheng, J., Xie, J., Jiang, D., Hsiang, T., and Fu, Y. 2016. A HOPS protein, CmVps39, is required for vacuolar morphology, autophagy, growth, conidiogenesis and mycoparasitic functions of Coniothyrium minitans. Environ. Microbiol. 18:3785-3797.

Yu, F., Gu, Q., Yun, Y., Yin, Y., Xu, J. R., Shim, W. B., and Ma, Z. 2014. The TOR signaling pathway regulates vegetative development and virulence in Fusarium graminearum. New Phytol. 203:219-232.

Yun, Y., Liu, Z., Zhang, J., Shim, W. B., Chen, Y., and Ma, Z. 2014. The MAPKK FgMkk1 of Fusarium graminearum regulates vegetative differentiation, multiple stress response, and virulence via the cell wall integrity and high-osmolarity glycerol signaling pathways. Environ. Microbiol. 16:2023-2037.

Zhang, H., Li, B., Fang, Q., Li, Y., Zheng, X., and Zhang, Z. 2016. SNARE protein FgVam7 controls growth, asexual and sexual development, and plant infection in Fusarium graminearum. Mol. Plant Pathol. 17:108-119.

Zhang, X. W., Jia, L. J., Zhang, Y., Jiang, G., Li, X., Zhang, D., and Tang, W. H. 2012. In planta stage-specific fungal gene profiling elucidates the molecular strategies of Fusarium graminearum growing inside wheat coleoptiles. Plant Cell 24:5159-5176.

Zheng, H., Zheng, W., Wu, C., Yang, J., Xi, Y., Xie, Q., Zhao, X., Deng, X., Lu, G., Li, G., Ebbole, D., Zhou, J., and Wang, Z. 2015. Rab GTPases are essential for membrane trafficking-dependent growth and pathogenicity in Fusarium graminearum. Environ. Microbiol. 17:4580-4599.

Zheng, W., Zheng, H., Zhao, X., Zhang, Y., Xie, Q., Lin, X., Chen, A., Yu, W., Lu, G., Shim, W. B., Zhou, J., and Wang, Z. 2016. Retrograde trafficking from the endosome to the trans-Golgi network mediated by the retromer is required for fungal development and pathogenicity in Fusarium graminearum. New Phytol. 210:1327-1343.

Zhou, X., Li, G., and Xu, J. R. 2011. Efficient approaches for generating GFP fusion and epitope-tagging constructs in filamentous fungi. Methods Mol. Biol. 722:199-212.

Zhou, X., Zhang, H., Li, G., Shaw, B., and Xu, J. R. 2012. The Cyclaseassociated protein Cap1 is important for proper regulation of infectionrelated morphogenesis in Magnaporthe oryzae. PLoS Pathog. 8: e1002911. 